\title{
Binary systems with post-T Tauri secondaries ${ }^{\star, \star \star}$
}

\author{
M. Gerbaldi ${ }^{1,2}$, R. Faraggiana ${ }^{3}$, and N. Balin ${ }^{4}$ \\ 1 Institut d'Astrophysique, 98 bis Bd. Arago, 75014 Paris, France \\ 2 Université de Paris Sud-XI, France \\ 3 Dipartimento di Astronomia, Università degli Studi di Trieste, Via G.B. Tiepolo 11, 34131 Trieste, Italy \\ e-mail: faraggiana@ts.astro.it \\ ${ }^{4}$ Institut National des Sciences Appliquées, Département de Mathématiques, 135 Av. de Rangueil, \\ 31077 Toulouse Cedex, France
}

Received 5 July 2000 / Accepted 2 August 2001

\begin{abstract}
The identification of post-T Tauri (pTT) stars selected through spectroscopic criteria by Pallavicini et al. (1992) and by Martín et al. (1992) among the candidates belonging to visual binary systems is revisited in the present paper by studying their position in the HR diagram. These stars belong to the so-called Lindroos binary sample (Lindroos 1985), i.e. to systems with early-type primaries and late-type companions. If these binaries are physical and not simply optical pairs, similar ages must be found for the early-type primary and the late-type component of each system. The ages of these systems have been derived by Lindroos in 1986, by using calibrations of the $u v b y \beta$ indices. In this paper, we revisit these ages through the position of these stars among new evolutionary tracks in the HR diagram for pre- and post-main sequence stars. We derive new estimations of the ages of each system component, as well as their masses, using parallaxes of the early-type component derived from Hipparcos data and by forcing the late-type companion to be at the same distance. $T_{\text {eff }}$ and $\log g$ of the early-type components have been computed using the calibrations of two independent photometric systems: the uvby $\beta$ photometry and the Geneva system. The $T_{\text {eff }}$ of the late-type stars have been determined by using various calibrations of several photometric systems: $u v b y \beta, U B V$ and $V R I$, in order to determine the uncertainties and systematic errors on these parameters and consequently on the ages. Differences in the ages and masses obtained by using various sources of recent evolutionary models are considered and discussed. The consistency of the age of the late type component with that of its early type primary is examined; the validity of this criterion for a selection of physical pairs is discussed. The accuracy of the observational and theoretical data are not sufficient to assign stringent values to the age for several of the examined systems. Nevertheless, in spite of the large error bars, we have established that we could select a number of systems which, according to their position in the HR diagram, may be physically associated. The selection of possible physically bounded systems obtained with the present approach and that made by Pallavicini et al. (1992) or Martín et al. (1992) on the basis of spectroscopic criteria are not always coincident. Spectroscopic criteria, for example the presence of a strong Li feature, are more stringent conditions than that of coherent ages of primaries and secondaries; however the Li I 6708 doublet is expected to fade in the latest stages of the pre-main sequence life of a star, so that the "oldest" pTTs may not be detected by spectroscopy only. The different results so obtained are discussed for each system and we conclude that the present approach may be used as a powerful criterion to select new pTT candidates in visual binaries to be observed and analyzed with high resolution spectrographs and to select candidates that have almost reached the main sequence.
\end{abstract}

Key words. binaries: visual - stars: evolution - stars: fundamental parameters (temperature, luminosity, age, mass) - Hertzsprung-Russel (HR) diagram - stars: pre-main sequence.

\author{
Send offprint requests to: M. Gerbaldi, \\ e-mail: gerbaldi@iap.fr \\ * Partly based on data from the ESA Hipparcos astrometric \\ satellite.

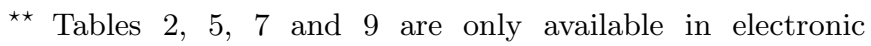 \\ form at the CDS via anonymous ftp to cdsarc.u-strasbg.fr \\ (130.79.128.5) or via \\ http://cdsweb.u-strasbg.fr/cgi-bin/qcat?J/A+A/379/162
}

\section{Introduction}

Post-T Tauri stars (pTTS) are pre-main sequence objects with masses similar to those of $\mathrm{T}$ Tauri stars, but older and so closer to the main sequence. The pTT stars do not show photometric and spectroscopic signatures of youth that characterize the classical $\mathrm{T}$ Tauri stars; only some weak remnant of line emission, IR excess and irregular 
variability are detectable, but at least the presence of the strong Li I 6708 resonance doublet remains as a characteristic of all the real pTTS, since Li destruction through convective mixing continues, in these stars, up to the early stages of their main sequence life. A strong Li I resonance line is also observed in the evolved stars, in post-main sequence phase (see references for example in Pilachowski et al. 2000) but these stars can be easily distinguished from the pTTs through their higher luminosity.

The pTT stars are supposed to be more numerous, about one order of magnitude higher, according to Herbig (1978), than the classical T Tauri stars because their evolutionary phase is longer. The exceptions may be represented by very young regions where classical T Tauri stars are expected to be present, while the more evolved pTTS had not yet the time to be produced and also if discontinuous star formation occurred in some clouds (Herbig 1978).

The detection of the elusive pTTS in the general field was first discussed by Murphy (1969) and carried out by Gahm et al. (1983) who searched for visual binaries with early type primaries and late type secondaries. The idea was that the main sequence lifetime of the high mass component is comparable to the contraction time scale of solar type stars; so if the systems are physical, the late type secondaries should still be contracting to the ZAMS or have recently arrived on it. With this hypothesis Lindroos (1985, 1986) identified 78 such visual systems. In order to distinguish the physical from the optical systems through the position of the stars in the HR diagram, Lindroos (1985) determined the astrophysical parameters by using Strömgren photometry and low resolution spectroscopy. The coherence of the ages determined by using Hejlesen (1980) and Iben \& Talbot (1966) isochrones is then discussed by the same author (Lindroos 1986).

Two studies by Pallavicini et al. (1992) [hereafter PPR] and by Martín et al. (1992) [hereafter MMR] were aimed to analyse the spectroscopic properties of a sample of the Lindroos survey in order to identify the physical systems with genuine pTT components. PPR studied the Li I 6708, $\mathrm{H}_{\alpha}$ and the $\mathrm{Ca}$ II $\mathrm{H}$ and $\mathrm{K}$ features in 39 secondaries with spectral types later than F2. MMR added radial velocity measurements to the spectroscopic and photometric analysis of their selected sample of 14 stars. These two studies concluded that only for about $1 / 3$ of the analysed systems is the duplicity physical and that the secondaries are really in the the pTT evolutionary phase.

It should be noted that for some stars there are discrepancies on the conclusion reached by PPR and MMR, for example for HD 27638 and HD 127304.

Distances derived from the Hipparcos (ESA 1997) experiment and more accurate evolutionary track computations for pre- and post-main sequence stars became available in the last years; bearing this in mind, we are looking, in the present paper, for refined ages to distinguish physical from optical systems. It is timely to do such work due to new photometric observations and their calibrations, new stellar evolutionary models and Hipparcos parallaxes.
Using as a starting point the parallaxes of the primaries measured by Hipparcos, and by forcing the companion candidates to be at the same distance as the primaries, we derive a new estimation of the ages of each system component and discuss the consistency of these ages.

The evolutionary status of each system will be analysed through the position of the components in the HR diagram among evolutionary tracks. This requires the knowledge of $T_{\text {eff }}, \log g$ and the absolute magnitude.

In Sect. 3 we discuss the parameters of the early type stars and the extinction determination. In Sect. 4 the parameters of the late type stars are determined. In Sect. 5 the position of stars in the HR diagram is presented from which the age of the stars is derived. Uncertainties in the observed parameters and systematic errors in the various calibrations used are taken into account to derive these ages. Differences in the ages obtained by using various sources of evolutionary models are considered. The reliability of the physical nature of each system is analysed in Sects. 6 and 7 we discuss the rôle of the age determination as a criterion to select physical pairs among these systems.

\section{The analysed systems}

The list of the analysed systems is given in Table 1.

For all the systems we have kept the designations used by PPR, which are the Lindroos (1983) ones. Two stars deserve some comments. HD 36151X and HD 138800X are the designations given by Lindroos (1983), but in the Hauck \& Mermilliod catalogue (1998) they are both quoted as a $\mathrm{C}$ component. It should also be noted that the late-type secondary of HD 87901 referred as the B component is HD 87884.

In this table the systems are listed under various groups according to the spectroscopic properties derived by PPR. The three selected groups are: almost physical systems, possible physical systems and likely optical systems. We added a fourth group including the stars analysed only by MMR.

The column labels are self explanatory. We only draw attention to the fact that the suffix $\mathrm{AB}$ in Cols. 1 and 10 means that in the Strömgren or Geneva photometry only the combined colours have been measured. Four stars have been found to be photometrically variable (suffix $V$ in Cols. 1 or 10$)$.

We have retained in this Table the star HD 53755 analysed by MMR, but low weight will be assigned to the values of the parameters derived for this object having a photometric variability reported in uvby $\beta$ and Geneva photometries. According to the Bright Star Catalogue (1994) [hereafter BSC] the system comprises 4 components and its radial velocity is referred to as "SB". Extinction $E(B-V)=0.190$ corresponding to $E(b-y)=0.137$ is derived by Papaj et al. (1991), from TD1 colours (Thompson et al. 1978); we have derived a value of 0.148 for $E(b-y)$. 
Table 1. Photometric data and atmospheric parameters for the sample: the groups are those defined by PPR and MMR.

\begin{tabular}{|c|c|c|c|c|c|c|c|c|c|c|c|}
\hline \multirow{2}{*}{$\begin{array}{l}\text { HD } \\
(1)\end{array}$} & \multirow{2}{*}{$\begin{array}{l}V \\
(2)\end{array}$} & \multirow{2}{*}{$\begin{array}{c}(b-y) \\
(3)\end{array}$} & \multirow{2}{*}{$\begin{array}{l}m_{1} \\
(4)\end{array}$} & \multirow{2}{*}{$\begin{array}{l}c_{1} \\
(5)\end{array}$} & \multirow{2}{*}{$\begin{array}{r}\beta \\
(6)\end{array}$} & \multirow{2}{*}{$\begin{array}{c}E(b-y) \\
(7)\end{array}$} & \multicolumn{2}{|c|}{$\begin{array}{c}T_{\text {eff }} \quad \log g \\
(u v b y \beta))\end{array}$} & \multirow[b]{2}{*}{ (10) } & \multicolumn{2}{|c|}{$\begin{array}{l}T_{\text {eff }} \quad \log g \\
\text { (Geneva) }\end{array}$} \\
\hline & & & & & & & $(8)$ & (9) & & (11) & (12) \\
\hline \multicolumn{12}{|l|}{ I } \\
\hline $560 \mathrm{~A}$ & 5.556 & -0.027 & 0.131 & 0.832 & 2.815 & 0.005 & 11370 & 4.10 & $\mathrm{AB}$ & 11452 & 4.18 \\
\hline $560 \mathrm{~B}$ & 10.370 & 0.500 & 0.360 & 0.420 & & & 5237 & & & & \\
\hline $33802 \mathrm{~A}$ & 4.473 & -0.042 & 0.118 & 0.597 & 2.755 & 0.012 & 13190 & 4.10 & $\mathrm{AB}$ & 13104 & 4.12 \\
\hline 33802 B & 9.923 & 0.491 & 0.259 & 0.285 & 2.575 & & 5239 & & & & \\
\hline $38622 \mathrm{~A}$ & 5.280 & -0.067 & 0.090 & 0.307 & 2.655 & 0.017 & 17340 & 3.57 & $\mathrm{~A}$ & 16940 & 3.85 \\
\hline $38622 \mathrm{C}$ & 12.011 & 0.406 & 0.194 & 0.325 & 2.635 & & 5768 & & & & \\
\hline $90972 \mathrm{~A}$ & 5.585 & -0.023 & 0.129 & 0.862 & 2.827 & 0.007 & 11170 & 4.13 & $\mathrm{AB}$ & 11125 & 4.20 \\
\hline 90972 B & 9.654 & 0.384 & 0.215 & 0.325 & 2.580 & & 5842 & & & & \\
\hline $108767 \mathrm{~A}$ & 2.900 & -0.024 & 0.142 & 0.980 & 2.856 & -0.006 & 10460 & 3.99 & $\mathrm{~A}$ & 10358 & 4.11 \\
\hline $108767 \mathrm{~B}$ & 8.430 & 0.534 & 0.423 & 0.310 & 2.566 & & 4975 & & & & \\
\hline $113703 \mathrm{~A}$ & 4.703 & -0.067 & 0.103 & 0.377 & 2.715 & 0.009 & 15830 & 4.21 & $\mathrm{~A}$ & 15545 & 4.27 \\
\hline $113703 \mathrm{~B}$ & 10.8 & & & & & & {$[5163]^{b}$} & & & & \\
\hline $113791 \mathrm{~A}$ & 4.272 & -0.085 & 0.091 & 0.154 & 2.656 & 0.012 & 20790 & 4.11 & $\mathrm{~A}$ & 20144 & 4.22 \\
\hline $113791 \mathrm{~B}$ & 9.381 & 0.335 & 0.187 & 0.312 & 2.620 & & 6196 & & & & \\
\hline $129791 \mathrm{~A}$ & 6.915 & 0.035 & 0.139 & 0.969 & 2.867 & 0.052 & 10260 & 4.15 & $\mathrm{~A}$ & 10245 & 4.21 \\
\hline $143939 \mathrm{~A}$ & 6.980 & -0.092 & 0.229 & 0.815 & 2.840 & -0.072 & 11320 & 4.34 & $\mathrm{ABV}$ & 11317 & 4.24 \\
\hline 143939 B & 11.800 & 0.640 & 0.590 & 0.240 & & & 4447 & & & & \\
\hline \multicolumn{12}{|l|}{ II } \\
\hline $17543 \mathrm{AB}$ & 5.240 & -0.015 & 0.092 & 0.487 & 2.703 & 0.051 & 14500 & 3.79 & & & \\
\hline $17543 \mathrm{C}$ & 10.727 & 0.395 & 0.149 & 0.309 & 2.565 & & 6046 & & & & \\
\hline $27638 \mathrm{~A}$ & 5.402 & -0.022 & 0.140 & 0.955 & 2.840 & -0.002 & 10510 & 3.94 & $\mathrm{~A}$ & 10492 & 4.18 \\
\hline $27638 \mathrm{~B}$ & 8.430 & 0.378 & 0.212 & 0.319 & 2.623 & & 5812 & & & & \\
\hline $40494 \mathrm{~A}$ & 4.366 & -0.074 & 0.091 & 0.364 & 2.645 & 0.005 & 16100 & 3.23 & $\mathrm{~A}$ & 15944 & 3.73 \\
\hline $40494 \mathrm{~B}$ & 12.664 & 0.458 & 0.227 & 0.280 & 2.572 & & 5367 & & & & \\
\hline $53191 \mathrm{~A}$ & 7.742 & -0.023 & 0.135 & 0.876 & 2.840 & 0.004 & 11040 & 4.20 & $\mathrm{~A}$ & 11138 & 4.12 \\
\hline $53191 \mathrm{~B}$ & 11.754 & 0.425 & 0.198 & 0.304 & 2.598 & & 5563 & & & & \\
\hline $60102 \mathrm{~A}$ & 7.537 & 0.020 & 0.119 & 0.916 & 2.819 & 0.045 & 10790 & 3.91 & $\mathrm{~A}$ & 11036 & 4.05 \\
\hline $60102 \mathrm{~B}$ & 11.862 & 0.519 & 0.217 & 0.208 & 2.704 & & 5211 & & & & \\
\hline $77484 \mathrm{AB}$ & 8.019 & -0.003 & 0.147 & 0.966 & 2.556 & 0.014 & 10280 & $4.10^{*}$ & $\mathrm{AB}$ & 10231 & 4.24 \\
\hline $127304 \mathrm{~A}$ & 6.060 & -0.010 & 0.148 & 1.007 & 2.879 & 0.004 & 10090 & 4.11 & A & 9990 & 4.13 \\
\hline 127304 B & 11.13 & & & & & & {$[5120]^{b}$} & & & & \\
\hline \multicolumn{12}{|l|}{ III } \\
\hline $8803 \mathrm{~A}$ & 6.643 & -0.006 & 0.094 & 0.885 & 2.821 & 0.027 & 11110 & 4.04 & $\mathrm{AB}$ & 11419 & 4.22 \\
\hline 8803 B & 9.670 & 0.310 & 0.260 & 0.730 & 2.685 & & 6686 & & & & \\
\hline $23793 \mathrm{AB}$ & 5.088 & -0.056 & 0.096 & 0.353 & 2.691 & 0.023 & 16420 & 3.97 & $\mathrm{AB}$ & 16021 & 4.20 \\
\hline 35007 A & 5.670 & -0.047 & 0.094 & 0.290 & 2.679 & 0.037 & 17730 & 4.01 & $\mathrm{~A}$ & 17130 & 4.23 \\
\hline $35007 \mathrm{C}$ & 11.882 & 0.435 & 0.205 & 0.372 & 2.625 & & 5733 & & & & \\
\hline $36013 \mathrm{~A}$ & 6.894 & -0.064 & 0.093 & 0.344 & 2.669 & 0.016 & 16640 & 3.67 & $\mathrm{~A}$ & 16117 & 3.97 \\
\hline 36013 B & 12.486 & 0.372 & 0.149 & 0.334 & 2.600 & & 5982 & & & & \\
\hline $36151 \mathrm{~A}$ & 6.695 & -0.041 & 0.098 & 0.383 & 2.705 & 0.034 & 15870 & 4.08 & $\mathrm{~A}$ & 15317 & 4.31 \\
\hline $36151 \mathrm{~B}$ & 10.597 & 0.411 & 0.180 & 0.362 & 2.565 & & 5859 & & & & \\
\hline $36151 \mathrm{X}$ & 11.988 & 0.467 & 0.106 & 0.353 & 2.600 & & 5515 & & & & \\
\hline 36779 A & 6.231 & -0.071 & 0.094 & 0.140 & 2.649 & 0.026 & 21280 & 4.05 & $\mathrm{~A}$ & 20616 & 4.20 \\
\hline 36779 B & 11.190 & 0.890 & 0.440 & 0.398 & 2.568 & & 3722 & & & & \\
\hline $43286 \mathrm{~A}$ & 6.994 & -0.055 & 0.105 & 0.421 & 2.686 & 0.016 & 15340 & 3.70 & $\mathrm{~A}$ & 14950 & 4.15 \\
\hline $43286 \mathrm{~B}$ & 12.381 & 0.423 & 0.258 & 0.248 & 2.592 & & 5617 & & & & \\
\hline $63465 \mathrm{AB}$ & 5.087 & -0.028 & 0.072 & 0.307 & 2.634 & 0.057 & 17260 & 3.24 & $\mathrm{AB}$ & 17275 & 3.70 \\
\hline 70309 A & 6.456 & -0.057 & 0.102 & 0.313 & 2.685 & 0.024 & 17200 & 4.01 & A & 16542 & 4.19 \\
\hline 70309 B & 11.282 & 0.700 & 0.387 & 0.263 & 2.604 & & 4310 & & & & \\
\hline $71510 \mathrm{~A}$ & 5.186 & -0.080 & 0.093 & 0.294 & 2.665 & 0.005 & 17580 & 3.77 & $\mathrm{~A}$ & 17015 & 4.10 \\
\hline $71510 \mathrm{C}$ & 10.766 & 0.420 & 0.237 & 0.383 & 2.600 & & 5639 & & & & \\
\hline
\end{tabular}


Table 1. continued.

\begin{tabular}{|c|c|c|c|c|c|c|c|c|c|c|c|}
\hline HD & $V$ & $(b-y)$ & $m_{1}$ & $c_{1}$ & $\beta$ & $E(b-y)$ & \multicolumn{2}{|c|}{$\begin{array}{l}T_{\text {eff }} \quad \log g \\
(u v b y \beta)\end{array}$} & & \multicolumn{2}{|c|}{$\begin{array}{l}T_{\text {eff }} \quad \log g \\
\text { (Geneva) }\end{array}$} \\
\hline (1) & (2) & (3) & (4) & (5) & (6) & (7) & (8) & (9) & (10) & (11) & (12) \\
\hline \multicolumn{12}{|l|}{ III } \\
\hline $76566 \mathrm{AV}$ & 6.264 & -0.072 & 0.098 & 0.334 & 2.667 & 0.008 & 16810 & 3.67 & AV & 16429 & 4.01 \\
\hline 76566 B & 12.639 & 0.419 & 0.238 & 0.287 & 2.601 & & 5613 & & & & \\
\hline $86388 \mathrm{~A}$ & 6.871 & -0.033 & 0.122 & 0.827 & 2.823 & 0.001 & 11350 & 4.18 & $\mathrm{AB}$ & 11594 & 4.27 \\
\hline 86388 B & 9.980 & 0.298 & 0.163 & 0.554 & 2.704 & & 6500 & & & & \\
\hline $87901 \mathrm{~A}$ & 1.355 & -0.037 & 0.102 & 0.712 & 2.723 & 0.010 & 12350 & 3.48 & A & 12285 & 3.82 \\
\hline 87901 B & 8.080 & & & & & & {$[4885]^{a}$} & & & & \\
\hline $106983 \mathrm{~A}$ & 4.026 & -0.084 & 0.103 & 0.259 & 2.683 & 0.002 & 18170 & 4.15 & A & 17656 & 4.22 \\
\hline 106983 B & 12.490 & 0.931 & 0.336 & 0.299 & 2.583 & & 3832 & & & & \\
\hline $112244 \mathrm{~A}$ & 5.388 & 0.080 & 0.018 & -0.137 & 2.538 & & & & & & \\
\hline 112244 B & 11.768 & 0.815 & 0.235 & 0.014 & 2.828 & & & & & & \\
\hline $123445 \mathrm{~A}$ & 6.191 & -0.016 & 0.109 & 0.746 & 2.801 & 0.026 & 11930 & 4.19 & A & 11991 & 4.28 \\
\hline $123445 \mathrm{~B}$ & 12.517 & 0.690 & 0.471 & 0.472 & 2.573 & & 4464 & & & & \\
\hline $127971 \mathrm{~A}$ & 5.883 & -0.038 & 0.117 & 0.578 & 2.757 & 0.018 & 13350 & 4.17 & A & 13361 & 4.22 \\
\hline 127971 B & 11.215 & 0.640 & 0.397 & 0.362 & 2.620 & & 4582 & & & & \\
\hline 137387 AV & 5.471 & -0.012 & 0.037 & 0.155 & 2.491 & 0.091 & 21010 & $3.72^{* *}$ & $\mathrm{AV}$ & 22750 & 3.79 \\
\hline 138800 A & 5.650 & 0.004 & 0.095 & 0.686 & 2.718 & 0.053 & 12610 & 3.50 & A & 12646 & 3.86 \\
\hline $138800 \mathrm{X}$ & 12.860 & 0.600 & 0.330 & 0.460 & & & 4970 & & & & \\
\hline $162082 \mathrm{~A}$ & 8.159 & 0.084 & 0.055 & 0.500 & 2.738 & 0.152 & 14440 & 4.24 & & & \\
\hline 162082 B & 11.054 & 0.374 & 0.124 & 0.625 & 2.772 & & 7098 & & & & \\
\hline $180183 \mathrm{~A}$ & 6.815 & -0.073 & 0.089 & 0.190 & 2.654 & 0.021 & 19890 & 3.95 & $\mathrm{~A}$ & 19787 & 4.19 \\
\hline 180183 B & 11.447 & 0.617 & 0.340 & 0.711 & 2.556 & & 4883 & & & & \\
\hline \multicolumn{12}{|l|}{ IV } \\
\hline $\begin{array}{l}53755 \mathrm{ABV} \\
53755 \mathrm{~B}\end{array}$ & $\begin{array}{c}6.494 \mathrm{~V} \\
10.300\end{array}$ & 0.030 & 0.020 & -0.011 & 2.585 & 0.148 & $\begin{array}{l}24610 \\
{[6410]^{a}}\end{array}$ & 3.51 & $\mathrm{ABV}$ & 27794 & 3.93 \\
\hline $174585 \mathrm{~A}$ & 5.900 & -0.051 & 0.074 & 0.253 & 2.661 & 0.039 & 18560 & 3.86 & $\mathrm{~A}$ & 17940 & 4.09 \\
\hline $174585 \mathrm{~B}$ & 10.890 & & & & & & {$[4790]^{a}$} & & & & \\
\hline $174585 \mathrm{C}$ & 10.300 & & & & & & {$[5955]^{a}$} & & & & \\
\hline
\end{tabular}

I) Pallavicini et al. (1992). Group a, almost certainly physical systems.

II) Pallavicini et al. (1992). Group b, possible physical systems.

III) Pallavicini et al. (1992). Group c, likely optical systems.

IV) Stars observed only by Martín et al. (1992).

* HD 77484: with the observed value of $\mathrm{H}_{\beta}, T_{\text {eff }}=8450 \mathrm{~K}$ and $\log g=0.66$; the given parameters have been computed disregarding the $\mathrm{H}_{\beta}$ value.

** HD 137387: the values of $T_{\text {eff }}$ and $\log g$ can be obtained only if the low value of the $\mathrm{H}_{\beta}$ index is disregarded.

${ }^{a} T_{\text {eff }}$ from MMR.

${ }^{b} T_{\text {eff }}$ from calibration of $(B-V)$ by Alonso et al. (1996a).

\section{3. eff and log of early-type stars}

For the brightest component of the systems, the temperature has been computed using the calibrations of two independent photometric systems: the uvby $\beta$ photometry and the Geneva system. These calibrations based on normal star data, have been adopted for all the primaries, in spite of the fact that two stars are classified Ap (HD 90972 and HD 143939) and one Be (HD 137387).

\subsection{The uvby $\beta$ photometry}

The photometric data and their errors are taken from the Hauck \& Mermilliod catalogue (1998).
The measurements refer to the A component alone except for the following stars: HD 77484AB, HD 23793AB, $\mathrm{HD} 63465 \mathrm{AB}$ and $\mathrm{HD} 53755 \mathrm{AB}$ with a companion located at $4 . .4,9 . \prime 0,100^{\prime \prime} 9$ and $6 .{ }^{\prime \prime} 6$ respectively. Other combined colour indices are those of HD 17543AB which has a B companion at 3 ". 2 with $V=8.8$ and another weak one recently discovered by Richichi et al. (1996) at 0"'04. All these companions have a magnitude difference of $3 \mathrm{mag}$ or more, so that no contamination is expected on the bright star components. We could test the degree of contamination on HD 8803 for which we have the values for HD 8803A and HD 8803AB (see Sects. 3.1.2 and 4.2).

For three other systems in the same separation range, namely HD 560, HD 86388 and HD 162082, the photometric colour indices have been measured separately for 
the two components; the contamination of visual photometric colours by the cool companion can be easily neglected since the magnitude differences for these three objects range from 2.9 to $4.1 \mathrm{mag}$ in $V$.

HD 90972A is known to be SB2 (BSC), therefore the photometric indices are physically meaningful only under the hypothesis that the two stars are very similar.

\subsubsection{Interstellar extinction}

Most of the early type stars are bright and a low interstellar extinction is expected. We computed the reddening using the program by Moon (1985) for all but HD 112244, which, being classified O9 Iab, lies outside the Moon calibration limits.

A fine analysis of the extinction versus the stellar distance and coordinates has been done in order to detect possible anomalies. The $E(b-y)$ values versus the distances derived from the Hipparcos parallaxes have been compared with extinction model expectations (Chen et al. 1998; Vergely et al. 1998). The adopted models predict different extinction curves for distance values larger than $200 \mathrm{pc}$, and we note a better agreement with the Chen et al. extinction law.

We computed the reddening difference between the observed and the Chen et al. predictions for each star. One star has a $E(b-y)$ difference higher than $|0.08|$ : HD 143939, with a high negative value of $E(b-y)$ indicating a non normal energy flux distribution. This characteristic is confirmed by its ultraviolet flux measured by the S2/68 spectrophotometers on board the TD1 satellite (Thompson et al. 1978), which, at wavelengths shorter than $2000 \AA$, is lower than expected because the differences between the observed and the computed values (based on Kurucz models) of $m_{\mathrm{UV}}-V$ are equal to -0.20 and -0.5 at 1965 and $1565 \AA$ respectively. We give low weight to the $-0.3 \mathrm{mag}$ found for $m_{\mathrm{UV}}-V$ at $2740 \AA$ because the underestimation of stellar fluxes of hot stars measured by the S2/68 photometer at this wavelength (Faraggiana \& Malagnini 1984) is known. For three stars the $E(b-y)$ difference value lies between $|0.03|$ and $|0.04|$ : HD 127304, HD 129791 and HD 137387.

A test has been made on the abnormality of the stellar photometric indices in order to detect possible emission in $\mathrm{H}_{\beta}$; for each star, the same Moon program has been used to compute the predicted $\mathrm{H}_{\beta}$ index to be compared with the observed one. For HD 77484 a significant difference is obtained: the observed value being 2.556 , while the value coherent with the other indices is 2.863. For HD 137387 the low observed $\mathrm{H}_{\beta}$ value (2.491) is not consistent with the other stellar colours so that only the predicted value, 2.632 , is used to derive $T_{\text {eff }}$ and $\log g$. This star is known to be a Be star with strong emission in Balmer lines which explains the low value of the observed $\mathrm{H}_{\beta}$ index; this high colour excess may be related to this peculiarity.

The star HD 162082 has the highest extinction value: $E(b-y)=0.152$ but it has not been observed by
Hipparcos, so its reddening value cannot be estimated from the Chen et al. (1998) model. The $E(b-y)$ value may indicate that this star is a peculiar object or an undetected binary, as suggested also by its ultraviolet magnitudes computed from TD1 fluxes (Thompson et al. 1978).

\subsection{2. $T_{\text {eff }}$ and $\log g$}

We have adopted the Moon \& Dworetsky (1985) [hereafter $\mathrm{MD}$ ] calibration for $u v b y \beta$ to which we have implemented the corrections by Castelli (1991). The observed values of the photometric indices, the derived $E(b-y), T_{\text {eff }}$ and $\log g$ are given Table 1 (Cols. 7-9).

We tested the influence of a faint companion on the photometric indices by examining the parameters derived for HD 8803, a star for which the available data refer to either HD 8803A $(V=6.643)$ or HD 8803AB $(V=6.593)$. The computed values are respectively: $E(b-y)=0.027$ and $0.018, T_{\text {eff }}=11110$ and $11240 \mathrm{~K}, \log g=4.04$ and 4.09. The presence of the late type companion classified A8V by Davidson et al. (1987) or F6Vp by Lindroos (1985) does not affect the atmospheric parameters of the B9V primary while the bright companion affects the colours of the cooler one which is much fainter (see Sect. 4.2).

\subsection{Geneva photometry}

The values of the Geneva photometry indices were retrieved from the Data Base on the Web site of the Geneva Observatory: General Catalogue of Photometric Data for the Geneva Photometric System by G. Burki et al., available electronically on the Web page of the Geneva Observatory (http://obswww.unige.ch/gcpd/ cgi-bin/photoSys.cgi).

Two stars are missing in this Data Base: HD 17543 and HD 162082. We recall that the percentage of AB measurements is higher in the Geneva than in the uvby $\beta$ photometry since only classical photometers which cannot separate stars closer than 10 arcsec have been used to collect these data.

The values of $T_{\text {eff }}$ and $\log g$ (Table 1 , Cols. 11 and 12) are obtained from the Künzli et al. (1997) calibration. No extinction value is required as an input to this calibration because reddening-free parameters are used to derive $T_{\text {eff }}$ and $\log g$ for the temperature range of these early-type stars.

HD 112244 is too hot to be calibrated by the Geneva program (Cramer 1993), as it is in the uvby $\beta$ photometry, so that this system will not be considered anyfurther.

\subsection{Accuracy of the parameters}

The errors on the determination of the parameters $T_{\text {eff }}$ and $\log g$ have two main origins: the uncertainties of the observations and those resulting from the calibrations of the photometric indices. 
The influence of the photometric indices accuracy on the determination of $T_{\text {eff }}$ and $\log g$ has been analysed for the subsample of stars whose errors of the $u v b y \beta$ colour indices are available. The extreme values of these indices, computed by adding and subtracting the observational errors, have been used to derive the corresponding values of $T_{\text {eff }}$ and $\log g$ which are given in Table 2. For each star, the half value of the difference between the two extreme values of these parameters constitutes the observational error.

For the stars with $T_{\text {eff }}$ less than $15000 \mathrm{~K}$ the mean value of these observational errors is $50 \mathrm{~K}$ with a standard deviation (std) of $35 \mathrm{~K}$; for stars with $T_{\text {eff }}$ larger than $15000 \mathrm{~K}$ but less than $20000 \mathrm{~K}$, the mean value is $72 \mathrm{~K}$ with an std of $57 \mathrm{~K}$. Only four stars are hotter than $20000 \mathrm{~K}$ : HD 36779, HD 113791, HD 137387 and HD 53775. We have already underlined the variability of HD 137387 and HD 53775; for the two other stars, even a small difference in the values of the photometric indices produces an important effect on $T_{\text {eff }}$, much larger than that found for cooler stars.

For the gravity parameter, $\log g$, the observational error has been computed with the same method; the mean error is 0.04 with an std of 0.04 .

A similar study cannot be undertaken for the Geneva photometry, the error of the photometric indices being not present in the General Catalogue of Photometric Data for the Geneva Photometric System.

The estimation of the error arising from the calibration process is not so straightforward. Castelli (1991) has studied the impact on $T_{\text {eff }}$ and $\log g$ of different calibrations of $c_{0}$ and $\mathrm{H}_{\beta}$ indices for a sample of non-supergiant B-type stars. She found that for stars in the temperature range of 10000 to $15000 \mathrm{~K}$, the $\Delta T_{\text {eff }}$ is of about $\pm 200 \mathrm{~K}$ and a higher uncertainty is expected for stars hotter than $20000 \mathrm{~K}$, probably from uncertainties on $\mathrm{H}_{\beta}$ index measurements.

The accuracy of the reddening value also plays a rôle in the value of $T_{\text {eff }}$. We have estimated the difference in $T_{\text {eff }}$ resulting from a difference of 0.01 mag in $E(b-y)$; this difference depends on the range of $T_{\text {eff }}$, it is larger for the hottest stars, and we shall take the largest computed one, that is $60 \mathrm{~K}$, as a representative value for this difference.

For the calibration of the Geneva photometric system, Künzli et al. (1997) estimates the error in $T_{\text {eff }}$ to be about $1 \%$, and that on $\log g$ to have a mean value of 0.15 .

Another way to estimate the accuracy of $T_{\text {eff }}, \log g$ is to compare the values of these parameters derived from these two photometric systems since their calibration methods are totally different.

We have computed the differences $\Delta T_{\text {eff }}$ and $\Delta \log g$ between the two sets of values, i.e. those derived from the MD calibration of the Strömgren indices minus those from the Künzli et al. calibration of the Geneva system. Two stars are not taken into consideration for such comparison: HD 53755 and HD 137387. HD 137387 is a Be star for which only an estimated value of $\mathrm{H}_{\beta}$ is accepted by the

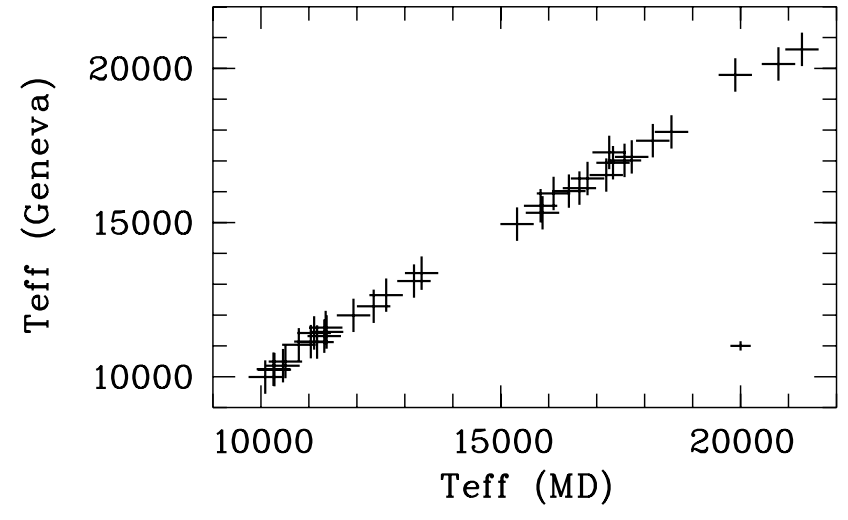

Fig. 1. Comparison of the temperature derived from the MD calibration of the uvby $\beta$ photometry and from the Geneva photometry with the Künzli et al. (1997) calibration. The stars HD 53755 and HD 137387 are not considered in this comparison. Typical error bars are plotted in the lower right corner.

Moon program; the variable HD 53755 has been discussed previously (Sect. 2).

Figures 1 and 2 display the relations between the values of $T_{\text {eff }}$ and $\log g$ derived from the Strömgren and Geneva photometries.

There is a systematic effect on the $T_{\text {eff }}$ determinations. From $\Delta T_{\text {eff }}$ values we evaluate a shift of $480 \mathrm{~K}$ between two temperature domains: less than $15000 \mathrm{~K}$ and greater than $15000 \mathrm{~K}$. For stars with a $T_{\text {eff }}$ lower than $15000 \mathrm{~K}$ the mean value of the difference is $-35 \mathrm{~K}$ with an std of $125 \mathrm{~K}$; for stars with $T_{\text {eff }}$ higher than $15000 \mathrm{~K}$ the mean value of $\Delta T_{\text {eff }}$ is $440 \mathrm{~K}$ with a std of $205 \mathrm{~K}$. We checked that this shift is not due to the choice of our sample but is also present when comparing the $T_{\text {eff }}$ obtained from these two calibrations for another star sample: the one used by Castelli (1991). From this larger sample we derive a smooth trend rather than simply a shift. The goal of this paper not being a complete comparison between these two calibrations we shall retain here simply the shift found for our sample of stars.

From Fig. 2 a systematic trend on $\log g$ appears, but it depends very slightly on $T_{\text {eff }}$; the $\log g$ (Gen) are systematically higher than those derived from $u v b y \beta$ photometry and the difference decreases towards high $\log g$ values. The origin of this effect is not explained. We checked this by applying the correction suggested by Napiwotzki et al. (1993). Such an effect has little consequence, due to the fact that, in this temperature range, the $T_{\text {eff }}$ dependence on gravity is very small (Castelli 1991).

Finally, any calibration has to be checked by comparing the derived data with those obtained for some stars but using fundamental methods. For the $\log g$ values, the data obtained from the analysis of the eclipsing binary systems are used in addition to those computed from stellar atmosphere models. Such data are in fact used by MD and Künzli et al. (1997) in their calibration process. As we do not use this parameter we shall not discuss this point further. 


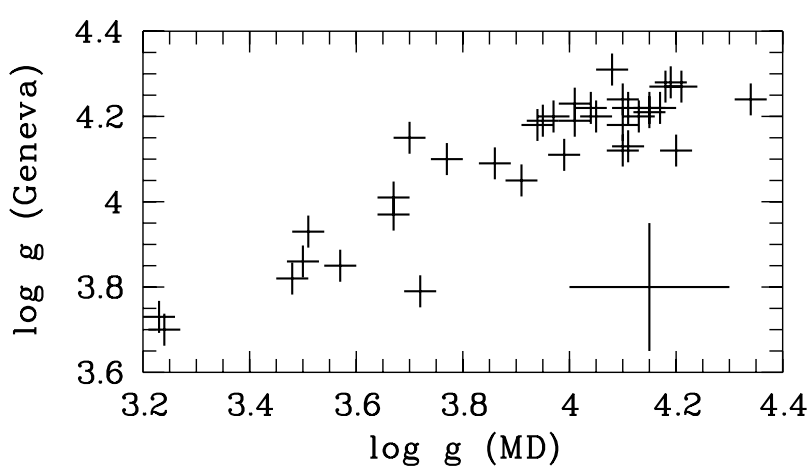

Fig. 2. Comparison of the gravities derived from the MD calibration of the uvby $\beta$ photometry and from the calibration of the Geneva photometry, using the Künzli et al. (1997) calibration. Typical error bars are plotted in the lower right corner.

The effective temperature can be determined directly only for a very small number of stars due to the lack of measurements of angular diameters and bolometric fluxes for hot stars. The InfraRed Flux Method (IRFM) (Blackwell et al. 1990) is the one used to derived the effective temperature; unfortunately, this method is less appropriate for hot stars because they have a large and uncertain ultra-violet contribution to the integrated flux, and moreover the determination of the interstellar extinction is less reliable in this spectral region. So, for the $T_{\text {eff }}$ calibration we cannot rule out the hypothesis that there is an offset that we cannot quantify in the calibration of each photometric system. We have simply shown the existence of a relative offset between the two used calibrations.

The adopted total error on $T_{\text {eff }}$ is the quadratic sum of the errors resulting from the photometric observations, that from the determination of $E(b-y)$, and those from the adopted calibrations of the chosen colour indices. We have computed the observational error on $T_{\text {eff }}$ for most of the stars of the sample. For the targets for which this error has not been computed, we take as the value $72 \mathrm{~K}$ which is the maximum of the two mean values of the observational errors computed for the other stars.

For the error resulting from the determination of the reddening $E(b-y)$ we adopt the value of $60 \mathrm{~K}$ as determined above.

For the error arising from the calibrations we shall take the value computed from the comparison between the calibrations of the Strömgren and the Geneva photometric systems, that is $125 \mathrm{~K}$ if $T_{\text {eff }}$ is lower than $15000 \mathrm{~K}$ and $205 \mathrm{~K}$ for higher values of the temperature. This systematic shift between the two temperature scales will be considered in the computation of stellar ages in Sect. 5 .

For each star the value of the total estimated error on $T_{\text {eff }}$ is given in Table 2. For $\log g$ we give only the observational error; this parameter is not used in the present analysis and the error on $\log g$ arising from the adopted calibrations has not been evaluated.

\section{Parameters of the late-type companions}

The best photometric systems to determine $T_{\text {eff }}$ for late type stars are those based on observations in the red or infra-red part of the spectrum, but only nine late-type companions of the sample have observations in the VRI Cousins system. So we have used also the available data from visual photometries and their relevant calibrations. Most of the observations of these targets were made in the $u v b y \beta$ and $U B V$ systems; no observations in the Geneva system have been performed on these targets. $T_{\text {eff }}$ and $\log g$ of the late-type companions computed from several calibrations of the quoted photometric systems are given below and will allow us to determine an error bar on such determinations.

\subsection{Calibration of uvby $\beta$ photometry}

For $\mathrm{G}$ and $\mathrm{K}$ types, the MD calibration is not as reliable as that obtained for the early type stars and does not extend below $T_{\text {eff }}=5500 \mathrm{~K}$; only a preliminary value of $T_{\text {eff }}$ is estimated by the Moon (1985) extinction program. Alonso et al. (1996a) provide a calibration of $T_{\text {eff }}$ from Strömgren photometric indices $(b-y)$ and $c_{1}$ for the low main sequence (F0V-K5V) stars and of $(b-y),(u-b)$ for the giants (Alonso et al. 1999). Only two objects are classified as giant: HD 106938B and 53755B, but only the first one has values in $u v b y \beta$.

For HD 106983, we note the incoherent value between the $T_{\text {eff }}(3832 \mathrm{~K})$ so derived and the spectral type G8 III given by Gahm et al. (1983).

\subsection{Calibration of UBV photometry}

Twelve stars have been observed in $U B V$, the data taken from the General Catalogue of Photometric Data by J.-C. Mermilliod, B. Hauck, M. Mermilliod are available electronically from the Web page of the Geneva observatory http://obswww. unige.ch/gcpd/gcpd.html. The calibrations used are those by Alonso et al. (1996a), by Houdashelt et al. (2000) and by Sekiguchi \& Fukugita (2000). The calibration of $T_{\text {eff }}$ in Sekiguchi \& Fukugita (2000) is computed for main sequence stars (Table 3 ) from the $(B-V)$ index by assuming a fixed $\log g$ value of 4.3 , that in Alonso et al. (1996a) is valid for dwarfs and that in Houdashelt et al. (2000) is given for a grid of $\log g$.

The star HD $8803 \mathrm{~B}$ has a $(B-V)$ value which does not fit the validity domain of any of these calibrations; the stars HD 560B and HD 8803B have too blue values for $(U-V)$ to allow the use of the Houdashelt et al. (2000) (Table 6) calibration. Since the cool components of HD 560 and HD 8803 have a small angular separation with their primaries ( $7{ }^{\prime \prime} 7$ and $6{ }^{\prime \prime} 0$ respectively), the contamination by the bright components is likely to be responsible for their anomalous $U B V$ colour indices. We remark also that for HD 560B, for which only one measure of the $U B V$ indices is available (Lutz \& Lutz 1977), the $V$ magnitude 9.44 is brighter than that quoted by PPR $(V=10.37)$. 
We note also that this star has shown a very large X-ray flare (Stelzer \& Huélamo 2000).

\subsection{Calibration of VRI photometry}

As calibrations of the $V R I$ indices for dwarf stars, we used the Alonso et al. (1996a) calibrations of $(V-R),(R-I)$ and $(V-I)$ to compute $T_{\text {eff }}$, as well as the Houdashelt et al. (2000) calibrations of $(V-R)$ and $(V-I)$ given in their Table 6 . We have transformed the Cousins colours into the corresponding ones in the Johnson system by using the relations given by Bessell (1983).

\section{4. $T_{\text {eff }}$ and $\log g$ for late-type companion stars}

All the photometric indices were corrected for the interstellar reddening using the value determined for the primary star; in case of negative $E(b-y)$ the star is considered unreddened. Classical relations were adopted for the various reddening coefficients, namely: $E(b-y)=1.35 E(B-V) ; E(U-B)=0.72 E(B-V) ;$ $E(V-R)=0.78 E(B-V) ; E(V-I)=1.60 E(B-V) ;$ $E\left(c_{1}\right)=0.195 E(b-y)$.

The $[\mathrm{Fe} / \mathrm{H}]$ values have been taken from MMR, when available; these values are small: their maximum and minimum are respectively +0.15 and -0.17 . For all the other stars we assume that their metallicity is solar because an $[\mathrm{Fe} / \mathrm{H}]$ value is computed, for the primaries, simultaneous with their $T_{\text {eff }}$ determination, using the Künzli et al. (1997) calibration of the Geneva photometric system, and the values so derived are all equal to 0.0. With our working hypothesis of physical systems, we have adopted the same value of 0.0 for the late-type companions. The consequence on the $T_{\text {eff }}$ values of the values chosen for $[\mathrm{Fe} / \mathrm{H}]$ is evaluated in the following section.

All these computed values for $T_{\text {eff }}$ are given in Table 3 where we added the $T_{\text {eff }}$ determined by MMR.

\subsection{Accuracy on $T_{\text {eff }}$ determination}

The determination of the accuracy on $T_{\text {eff }}$ is done through the estimation of the errors arising from the observations and of those from the calibrations including the errors on the interstellar reddening and on the $[\mathrm{Fe} / \mathrm{H}]$ determination.

As no estimation of error on the Strömgren photometric indices is given for these stars in the Hauck \& Mermilliod (1998) Catalogue, we cannot compute the corresponding observational error on $T_{\text {eff }}$.

For the photometric observations made in $U B V R I$ by MMR, the error bars are given, so we have estimated the induced error on $T_{\text {eff }}$, using the same method as for the early-type stars. For the 9 stars having such measurements, the mean error is of $140 \mathrm{~K}$ on the $T_{\text {eff }}$ value computed from Houdashelt et al. (2000) calibration of $(V-R)$. We shall adopt this value as the observational error for all the stars. We have checked that the use of the $(V-I)$ index to compute this error would have produced a similar result and this is the case with any other of the quoted calibrations of the VRI photometry.

We shall now evaluate the other sources of error entering into the computations.

The first one is the error on the reddening. We estimate it by adding arbitrarily 0.01 mag to $E(b-y)$ and computing the $T_{\text {eff }}$ using the Alonso et al. (1996a) calibration of Strömgren photometry. We choose the ubvy $\beta$ photometry to do this test because the largest number of stars is observed in this system. The mean value of the difference for 28 stars is $56 \mathrm{~K}$.

Another error may result from the adopted $[\mathrm{Fe} / \mathrm{H}]$ value. For the stars for which this parameter is given by MMR, we have computed the $T_{\text {eff }}$ by assuming the metallicity equal to solar. We did it using only the $(B-V)$ and $(V-R)$ calibrations by Alonso et al. (1996a) because there is no metallicity term in their calibration of the $(V-I)$ index. The error resulting from the $[\mathrm{Fe} / \mathrm{H}]$ choice is computed for the 6 stars having both $(B-V)$ and $[\mathrm{Fe} / \mathrm{H}]$ values: it is equal to $53 \mathrm{~K}$; for the 7 stars having both $(V-R)$ and $[\mathrm{Fe} / \mathrm{H}]$ values it is equal to $18 \mathrm{~K}$.

The source of errors arising from the calibration of the photometric indices can be estimated by comparing the calibrations used by each author. These calibrations have been obtained through various methods ranging from the IRFM (Alonso et al. 1996b; Sekiguchi \& Fukugita 2000) to the use of stellar atmospheric models (Houdashelt et al. 2000 ); the comparison of the $T_{\text {eff }}$ so obtained will give us an estimation of their accuracy.

The comparison between the $T_{\text {eff }}$ obtained from MD and Alonso et al. (1996a) calibrations of the Strömgren photometric system is displayed in Fig. 3; small systematic effects are present. The mean value of the differences between these two $T_{\text {eff }}$ is $14 \mathrm{~K}$ with an std of $125 \mathrm{~K}$. Due to the trend noticed in Fig. 3, we computed also the mean value for three domains in $T_{\text {eff. }}$ For $T_{\text {eff }}$ less than $5000 \mathrm{~K}$, the mean value of this difference is $-69 \mathrm{~K}$ with an std of $103 \mathrm{~K}$; for $T_{\text {eff }}$ in the domain $5000-5800 \mathrm{~K}$, the mean value of this difference is $8 \mathrm{~K}$ with an std of $105 \mathrm{~K}$ and for $T_{\text {eff }}$ larger than $5800 \mathrm{~K}$, the mean value of this difference is $93 \mathrm{~K}$ with an std of $118 \mathrm{~K}$. The stars HD 36151X and HD 43286B have differences in $T_{\text {eff }}$ larger than $400 \mathrm{~K}$, and they are not considered for the computations of the average values.

The comparison between the three calibrations of the $(B-V)$ indices present only a small systematic trend; as we shall not use these calibrations in the following section we do not discuss them in detail.

Two groups Alonso et al. (1996a) and Houdashelt et al. (2000) have defined several calibrations using the VRI photometry. For each star, we have computed the mean value of $T_{\text {eff }}$ determined from the colour indices $(R-I)$, $(V-R)$ and $(V-I)$ in the case of the first authors calibration and with $(V-R)$ and $(V-I)$ for the second one. We have checked that the differences between the $T_{\text {eff }}$, from different color indices, give a standard deviation of the same order as the one given in each of these papers. 
Table 3. $T_{\text {eff }}$ for late-type companions, determined from different calibrations of photometric indices 1: $T_{\text {eff }}$ from MD cali-

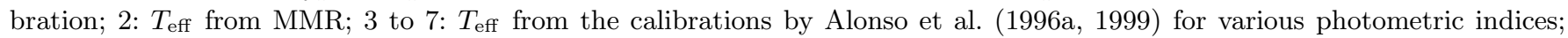
8 to 11: $T_{\text {eff }}$ from the calibrations by Houdashelt et al. (2000) for various photometric indices; 12 : $T_{\text {eff }}$ from Sekiguki \& Fukugita (2000) calibration of $(B-V)$.

\begin{tabular}{|c|c|c|c|c|c|c|c|c|c|c|c|c|c|}
\hline HD & & $\begin{array}{c}T_{\text {eff }} \\
1 \\
\mathrm{MD}\end{array}$ & $\begin{array}{c}T_{\text {eff }} \\
2 \\
\text { MMR }\end{array}$ & $\begin{array}{c}T_{\text {eff }} \\
3 \\
\text { Alonso } \\
B-V\end{array}$ & $\begin{array}{c}T_{\text {eff }} \\
4 \\
\text { et } \\
V-R\end{array}$ & $\begin{array}{c}T_{\text {eff }} \\
5 \\
\text { al. } \\
R-I\end{array}$ & $\begin{array}{c}T_{\text {eff }} \\
6 \\
(1996 \mathrm{a}) \\
V-I\end{array}$ & $\begin{array}{c}T_{\text {eff }} \\
7 \\
(1999) \\
b-y\end{array}$ & $\begin{array}{c}T_{\text {eff }} \\
8 \\
\text { Houdashelt } \\
U-V\end{array}$ & $\begin{array}{c}T_{\text {eff }} \\
9 \\
\text { et } \\
B-V\end{array}$ & $\begin{array}{c}T_{\text {eff }} \\
10 \\
\text { al. } \\
V-R\end{array}$ & $\begin{array}{c}T_{\text {eff }} \\
11 \\
(2000) \\
V-I\end{array}$ & $\begin{array}{c}T_{\text {eff }} \\
12 \\
\text { Sekiguchi \& Fukugita } \\
B-V\end{array}$ \\
\hline 560 & B & 5220 & 5370 & 7087 & 5531 & 5366 & 5419 & 5237 & - & 6846 & 5587 & 5526 & 7222 \\
\hline 33802 & B & 5310 & 5250 & - & 5489 & 5353 & 5374 & 5239 & - & - & 5560 & 5501 & - \\
\hline 38622 & $\mathrm{C}$ & 5950 & 5850 & 6122 & 5914 & 6333 & 6032 & 5768 & - & 6127 & 5938 & 6154 & 6292 \\
\hline 90972 & B & 6020 & - & - & - & - & - & 5842 & - & - & - & - & - \\
\hline 108767 & B & 4860 & 4930 & 5165 & 5114 & 4954 & 4987 & 4975 & 5087 & 5241 & 5161 & 5081 & 5360 \\
\hline 113703 & B & - & - & 5163 & - & - & - & - & - & 5273 & - & - & 5358 \\
\hline 113791 & B & 6330 & - & 6254 & - & - & - & 6196 & 6257 & 6242 & - & - & 6421 \\
\hline 129791 & B & - & - & - & - & - & - & - & - & - & - & - & - \\
\hline 143939 & B & 4470 & - & - & - & - & - & 4447 & - & - & - & - & - \\
\hline 17543 & $\mathrm{C}$ & 6210 & - & - & - & - & - & 6046 & - & - & - & - & - \\
\hline 27638 & B & 6010 & 5750 & 5858 & 5797 & 5797 & 5787 & 5812 & 5982 & 5949 & 5858 & 5864 & 6039 \\
\hline 40494 & B & 5440 & - & - & - & - & - & 5367 & - & - & - & - & - \\
\hline 53191 & B & 5590 & - & - & - & - & - & 5563 & - & - & - & - & - \\
\hline 60102 & B & 5330 & - & - & - & - & - & 5211 & - & - & - & - & - \\
\hline 77484 & B & - & - & - & - & - & - & - & - & - & - & - & - \\
\hline 127304 & B & - & - & 5120 & 5047 & 5132 & 5064 & - & 5137 & 5203 & 5175 & 5175 & 5316 \\
\hline 8803 & B & 6590 & - & - & - & - & - & 6686 & 6908 & - & - & - & - \\
\hline 23793 & B & - & - & - & - & - & - & - & - & - & - & - & - \\
\hline 35007 & $\mathrm{C}$ & 5730 & 5550 & 5695 & 5804 & 5729 & 5642 & 5733 & 5747 & 5756 & 5845 & 5837 & 5877 \\
\hline 36013 & B & 6140 & - & - & - & - & - & 5982 & - & - & - & - & - \\
\hline 36151 & B & 5610 & 5810 & - & - & - & - & 5859 & - & - & - & - & - \\
\hline 36151 & $\mathrm{X}$ & 6000 & - & - & - & - & - & 5515 & - & - & - & - & - \\
\hline 36779 & B & 3750 & 3990 & - & - & - & - & 3722 & - & - & - & - & - \\
\hline 43286 & B & 6010 & 5650 & 5844 & 5763 & 5587 & 5620 & 5617 & 5933 & 5888 & 5810 & 5750 & 6022 \\
\hline 63465 & B & - & - & - & - & - & - & - & - & - & - & - & - \\
\hline 70309 & B & 4350 & - & - & - & - & - & 4310 & - & - & - & - & - \\
\hline 71510 & $\mathrm{C}$ & 5640 & - & - & - & - & - & 5639 & - & - & - & - & - \\
\hline 76566 & B & 5660 & - & - & - & - & - & 5613 & - & - & - & - & - \\
\hline 86388 & B & 6500 & - & - & - & - & - & 6500 & - & - & - & - & - \\
\hline 87901 & B & - & 4885 & 4948 & 4985 & 4871 & 4914 & - & 5090 & 5122 & 5150 & 5024 & 5146 \\
\hline 106983 & B & 3570 & - & - & - & - & - & 3832 & - & - & - & - & - \\
\hline 123445 & B & 4390 & - & - & - & - & - & 4464 & - & - & - & - & - \\
\hline 127971 & B & 4540 & - & - & - & - & - & 4582 & - & - & - & - & - \\
\hline 137387 & B & - & - & - & - & - & - & - & - & - & - & - & - \\
\hline 138800 & $\mathrm{X}$ & 4810 & - & - & - & - & - & 4970 & - & - & - & - & - \\
\hline 162082 & B & 7020 & - & - & - & - & - & 7098 & - & - & - & - & - \\
\hline 180183 & B & 4630 & - & - & - & - & - & 4883 & - & - & - & - & - \\
\hline 53755 & B & - & 6410 & - & - & - & - & - & - & - & - & - & - \\
\hline 174585 & B & - & 4790 & 4669 & - & - & - & - & 4804 & 4822 & - & - & 4865 \\
\hline 174585 & $\mathrm{C}$ & - & 5955 & - & - & - & - & - & - & - & - & - & - \\
\hline
\end{tabular}

Between Alonso et al. (1996a) and Houdashelt et al. (2000) calibrations there is an offset of $96 \mathrm{~K}$.

Then we compared the differences between the $T_{\text {eff }}$ computed from the Alonso et al. (1996a) calibrations of Strömgren photometry and of the VRI ones; the mean value is equal to $107 \mathrm{~K}$ with an std of $128 \mathrm{~K}$ for the 7 stars in common between these two photometric systems.

Another comparison can be done between the $T_{\text {eff }}$ values computed from the mean $T_{\text {eff }}$ obtained from the Alonso et al. (1996a) calibrations of various colour indices of the VRI photometry and that obtained from the MD one of the uvby $\beta$ photometry. Seven dwarf stars are in common and the mean value of the differences in $T_{\text {eff }}$ is $6 \mathrm{~K}$ with an std of $213 \mathrm{~K}$; there is also a small systematic trend.

By combining all these comparisons, we derived the accuracy on the $T_{\text {eff }}$ determination of the late-type stars of the sample. The value is the same for all the stars: $266 \mathrm{~K}$; it is the quadratic sum of the following terms: $140 \mathrm{~K}$ for the error on the photometric observations, $56 \mathrm{~K}$ for the error 


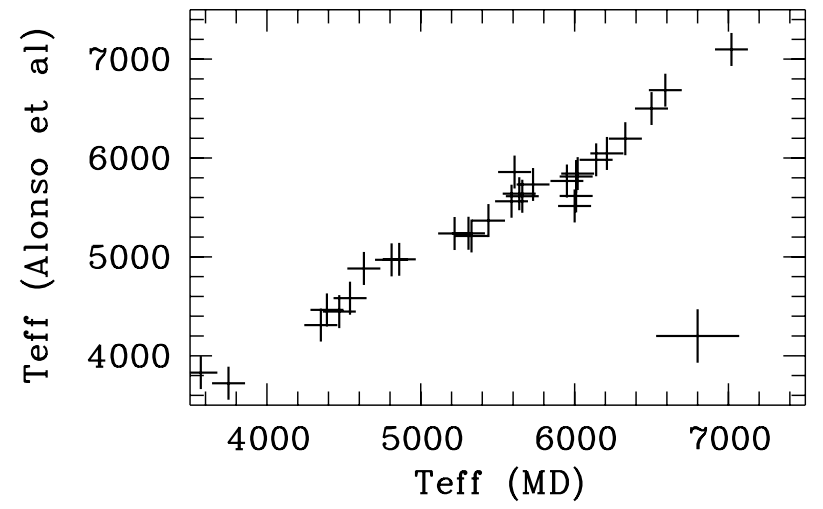

Fig. 3. Comparison of the temperature derived from the MD and the Alonso et al. (1996a) calibrations in $T_{\text {eff }}$ of the Strömgren photometric system. The $T_{\text {eff }}$ values are those given in Table 3, Cols. 1 and 7. Typical error bars are plotted in the lower right corner.

on the reddening, $53 \mathrm{~K}$ for the error on the metallicity, and $213 \mathrm{~K}$ for the error on the calibration of the photometric indices; in each case we retain the largest value.

As for the early type stars an offset in the $T_{\text {eff }}$ scale may be present. It could be pointed out by looking at the relations between the $T_{\text {eff }}$ obtained for the same star from different calibrations but it is difficult to quantify its value because the number of stars measured both in $V R I$ and Strömgren is small.

The $T_{\text {eff }}$ value we adopt for the age determination is that which we obtain from the Alonso et al. (1996a) calibration of $(b-y)$; this is the one given in Table 1 . We have selected such effective temperature determination because most of the stars have a $(b-y)$ value, which is not the case with other photometric indices and also because the $T_{\text {eff }}$ value obtained with this calibration do not present any systematic effect compared to other calibrations.

\section{HR diagram}

In order to construct the HR diagrams displayed in Figs. 4 and 5 we computed the distance of the system components by using the Hipparcos parallax of the brightest one, that observed by Hipparcos, and assuming that all these systems are physical. From the absolute magnitude $M_{V}$ (corrected for the reddening as explained in Sect. 2), the $M_{\text {bol }}$ has been derived by using the bolometric corrections taken from Bessell et al. (1998). The $L / L_{\odot}$ is obtained by adopting $M_{\text {bol } \odot}=4.75$ (1997, IAU General Assembly, Commissions 29 and 36) (Table 4, Cols. 5 and 6).

Before determining stellar ages the accuracy on the $L / L_{\odot}$ must be evaluated, that on $T_{\text {eff }}$ having been already discussed.

The errors on $L / L_{\odot}$ arise from the computation of $M_{\text {bol }}$, arising mainly from that on $M_{V}$ (we do not take into account any error on the bolometric correction):

$M_{V}=V+5+5 \log (\pi)-A v$.
The error on $M_{V}$ can be expressed as:

$\sigma(M v)=\left((\sigma(V))^{2}+(2.17 \sigma(\pi) / \pi)^{2}+(\sigma(A v))^{2}\right)^{0.5}$.

For the primaries, the $V$ magnitude and their error are given in the Hipparcos Catalogue; a constant value, of $\sigma(V)=0.01$, has been adopted by us. For the secondaries, the error on $V$ is known only for the stars studied by MMR; we adopted a constant value of 0.04 .

The value of $\sigma(\pi)$, the principal term on the budget error is taken from the Hipparcos Catalogue.

We adopt 0.05 as error on $A v$, since the error on $E(b-y)$ is about 0.01 . This value may be underestimated for the two reddened stars (HD 162082 and HD 53755). The value of $\sigma\left(\log \left(L / L_{\odot}\right)\right)$ is equal to $\sigma(M v) / 2.5$. We do not take into account any error on the determination of the bolometric magnitude for the Sun; what would play a role is the shift between the value adopted here and the one used in the computation of the evolutionary tracks.

The values of the parallax, $\sigma(\pi), M_{\mathrm{bol}}, \log \left(L / L_{\odot}\right)$ and $\log T_{\text {eff }}$, are given Table 4 .

In Table 5 , the values of $T_{\text {eff }}$ and $\log g$ are given; the errors are taken into account by giving for each parameter the minimum and maximum values.

\subsection{Ages and masses of the early-type stars}

Ages and masses are determined through interpolation among isochrones computed from various evolutionary models. The recent stellar evolutionary models with available isochrones are: Schaller et al. (1992), Meynet et al. (1993), Claret \& Gimenez (1992), updated by Claret (1995), Bertelli et al. (1994) updated by Girardi et al. (2000). The differences between these models are due mainly to the adopted value of the mixing length free parameter and to the opacity library used (Los Alamos Opacity Library (Huebner, W. F., Merts, A. L., Maage, N. H. Jr., Argo, M. F. (eds.) 1977, Astrophys. Opacity Library, UC-346) and Livermore Library (Rogers, F. J., Iglesias, C. A., 1992, ApJS, 79, 507)).

Values of masses and ages have been computed with the Schaller et al. (1992) evolutionary tracks and Meynet et al. (1993) isochrones and with the Girardi et al. (2000) evolutionary tracks and isochrones, assuming solar metallicity.

These values are given in Table 6 (Cols. 3, and 7 for ages and 5 and 9 for masses). Ages and masses computed with the two sets of theoretical grids are not significantly different, as shown in Figs. 6 and 7.

The errors on $T_{\text {eff }}$ and $\log L / L_{\odot}$ have been added and subtracted (error bars plotted in Fig. 4) to determine the error box on age and mass for each star. The extreme values of these parameters are given in Table 7 for each star, while in Cols. $(4,8)$ and $(6,10)$ of Table 6 the resulting uncertainties expressed in percentage are given for the ages and masses, for each set of models.

The label "ZAMS" in Tables 6 and 7 indicates a star located at the very beginning of an isochrone. 
Table 4. Stellar parameters for the sample stars; the parallaxes refer only to the primary star; Cols. 5-7 refer to the A components, Cols. $9-11$ to the secondaries.

\begin{tabular}{|c|c|c|c|c|c|c|c|c|c|c|}
\hline $\begin{array}{r}\text { Hip } \\
(1)\end{array}$ & $\begin{array}{r}\pi \\
(2)\end{array}$ & $\begin{array}{r}\sigma(\pi) \\
(3)\end{array}$ & $\begin{array}{r}\text { HD } \\
(4)\end{array}$ & $\begin{array}{r}M_{\mathrm{bol}} \\
(5)\end{array}$ & $\begin{array}{r}\log L / L_{\odot} \\
(6)\end{array}$ & $\begin{array}{r}\log T_{\text {eff }} \\
(7)\end{array}$ & $\begin{array}{r}\text { HD } \\
(8)\end{array}$ & $\begin{array}{r}M_{\text {bol }} \\
\quad(9)\end{array}$ & $\begin{array}{r}\log L / L_{\odot} \\
(10)\end{array}$ & $\begin{array}{r}\log T_{\text {eff }} \\
(11)\end{array}$ \\
\hline \multicolumn{11}{|l|}{ I } \\
\hline 813 & 10.04 & 0.86 & 560 & -0.01 & 1.90 & 4.06 & $560 \mathrm{~B}$ & 5.18 & -0.17 & 3.72 \\
\hline 24244 & 13.53 & 0.69 & 33802 & -0.81 & 2.23 & 4.12 & $33802 \mathrm{~B}$ & 5.35 & -0.24 & 3.72 \\
\hline 27364 & 4.08 & 0.86 & 38622 & -3.31 & 3.22 & 4.24 & $38622 \mathrm{C}$ & 4.92 & -0.07 & 3.76 \\
\hline 51376 & 6.78 & 0.74 & 90972 & -0.79 & 2.22 & 4.05 & 90972 B & 3.72 & 0.41 & 3.77 \\
\hline 60965 & 37.11 & 0.69 & 108767 & 0.44 & 1.73 & 4.02 & $108767 \mathrm{~B}$ & 5.99 & -0.49 & 3.70 \\
\hline 63945 & 7.88 & 0.72 & 113703 & -2.18 & 2.77 & 4.20 & $113703 \mathrm{~B}$ & 5.05 & -0.12 & 3.71 \\
\hline 64004 & 7.92 & 0.81 & 113791 & -3.33 & 3.23 & 4.32 & 113791 B & 3.80 & 0.87 & 3.79 \\
\hline 72192 & 7.72 & 0.94 & 129791 & 0.82 & 1.57 & 4.01 & 129791 B & & & \\
\hline 78756 & 5.97 & 0.95 & 143939 & 0.31 & 1.78 & 4.05 & 143939 B & 5.10 & -0.13 & 3.65 \\
\hline \multicolumn{11}{|l|}{ II } \\
\hline 13165 & 5.41 & 1.06 & 17543 & -2.40 & 2.86 & 4.16 & $17543 \mathrm{C}$ & 4.14 & 0.24 & 3.78 \\
\hline 20430 & 12.19 & 1.00 & 27638 & 0.45 & 1.72 & 4.02 & $27638 \mathrm{~B}$ & 3.80 & 0.38 & 3.76 \\
\hline 28199 & 3.82 & 0.54 & 40494 & -4.12 & 3.55 & 4.21 & $40494 \mathrm{~B}$ & 5.40 & -0.26 & 3.73 \\
\hline 33717 & 4.82 & 0.59 & 53191 & 0.64 & 1.64 & 4.04 & $53191 \mathrm{~B}$ & 5.04 & -0.12 & 3.74 \\
\hline 34753 & 4.84 & 0.57 & 60102 & 0.35 & 1.76 & 4.03 & 60102 B & 4.91 & -0.06 & 3.72 \\
\hline 44413 & 4.00 & 0.97 & 77484 & 0.66 & 1.64 & 4.01 & $77484 \mathrm{~B}$ & & & \\
\hline 70892 & 9.35 & 0.71 & 127304 & 0.62 & 1.65 & 4.00 & $127304 \mathrm{~B}$ & 5.95 & -0.48 & 3.71 \\
\hline \multicolumn{11}{|l|}{ III } \\
\hline 6751 & 6.25 & 0.97 & 8803 & -0.02 & 1.91 & 4.05 & 8803 B & 5.54 & 0.48 & 3.82 \\
\hline 17771 & 5.77 & 0.97 & 23793 & -2.64 & 2.96 & 4.22 & $23793 \mathrm{~B}$ & & & \\
\hline 25028 & 3.03 & 0.77 & 35007 & -3.70 & 3.38 & 4.25 & $35007 \mathrm{C}$ & 4.06 & 0.27 & 3.76 \\
\hline 25648 & 3.30 & 0.88 & 36013 & -2.03 & 2.71 & 4.22 & 36013 B & 4.97 & -0.09 & 3.78 \\
\hline 25712 & 2.70 & 0.81 & 36151 & -2.64 & 2.96 & 4.20 & $36151 \mathrm{~B}$ & 2.55 & 0.88 & 3.77 \\
\hline 25712 & 2.70 & 0.81 & 36151 & -2.64 & 2.96 & 4.20 & $36151 \mathrm{X}$ & 3.88 & 0.35 & 3.74 \\
\hline 26106 & 2.63 & 0.87 & 36779 & -3.86 & 3.44 & 4.33 & 36779 B & 1.87 & 1.15 & 3.57 \\
\hline 29713 & 3.25 & 0.84 & 43286 & -1.75 & 2.60 & 4.19 & $43286 \mathrm{~B}$ & 4.77 & -0.01 & 3.75 \\
\hline 38010 & 2.63 & 0.53 & 63465 & -4.62 & 3.75 & 4.24 & $63465 \mathrm{~B}$ & & & \\
\hline 40749 & 3.96 & 0.55 & 70309 & -2.21 & 2.78 & 4.24 & 70309 B & 3.46 & 0.52 & 3.63 \\
\hline 41296 & 4.84 & 0.45 & 71510 & -3.02 & 3.11 & 4.25 & $71510 \mathrm{C}$ & 4.08 & 0.27 & 3.75 \\
\hline 43807 & 3.49 & 0.61 & 76566 & -2.55 & 2.92 & 4.23 & $76566 \mathrm{~B}$ & 5.22 & -0.19 & 3.75 \\
\hline 48641 & 5.65 & 0.68 & 86388 & -0.04 & 1.92 & 4.05 & $86388 \mathrm{~B}$ & 3.74 & 0.41 & 3.81 \\
\hline 49669 & 42.09 & 0.79 & 87901 & -1.28 & 2.41 & 4.09 & 87901 B & 5.88 & -0.45 & 3.69 \\
\hline 60009 & 9.03 & 0.55 & 106983 & -2.87 & 3.05 & 4.26 & 106983 B & 5.93 & -0.47 & 3.58 \\
\hline 69113 & 4.57 & 0.76 & 123445 & -1.26 & 2.40 & 4.08 & $123445 \mathrm{~B}$ & 5.12 & -0.15 & 3.65 \\
\hline 71353 & 9.10 & 0.78 & 127971 & -0.31 & 2.02 & 4.13 & 127971 B & 5.43 & -0.27 & 3.66 \\
\hline 76013 & 3.20 & 0.59 & 137387 & -4.50 & 3.70 & 4.32 & $137387 \mathrm{~B}$ & & & \\
\hline 76750 & 4.44 & 0.57 & 138800 & -2.11 & 2.75 & 4.10 & $138800 \mathrm{X}$ & 5.58 & -0.33 & 3.70 \\
\hline 94899 & 4.10 & 0.80 & 180183 & -2.14 & 2.76 & 4.30 & 180183 В & 4.09 & 0.26 & 3.69 \\
\hline \multicolumn{11}{|l|}{ IV } \\
\hline 34234 & 0.92 & 0.880 & 53755 & -6.71 & 4.58 & 4.39 & $53755 \mathrm{~B}$ & -0.46 & 2.08 & 3.81 \\
\hline 92398 & 3.23 & 0.560 & 174585 & -3.44 & 3.27 & 4.27 & $174585 \mathrm{~B}$ & 2.97 & 0.71 & 3.68 \\
\hline 92398 & 3.23 & 0.560 & 174585 & -3.44 & 3.27 & 4.27 & $174585 \mathrm{C}$ & 2.64 & 0.84 & 3.77 \\
\hline
\end{tabular}

The age determination is ambiguous when the star position in the HR diagram corresponds to the end of the main sequence phase and so the star is located in a region where evolutionary tracks overlap. In such a case, a "**" in Tables 6 and 7 replaces the not determined value. For HD 53755 the error of $96 \%$ on the parallax prevents the computation of a reliable luminosity value. 


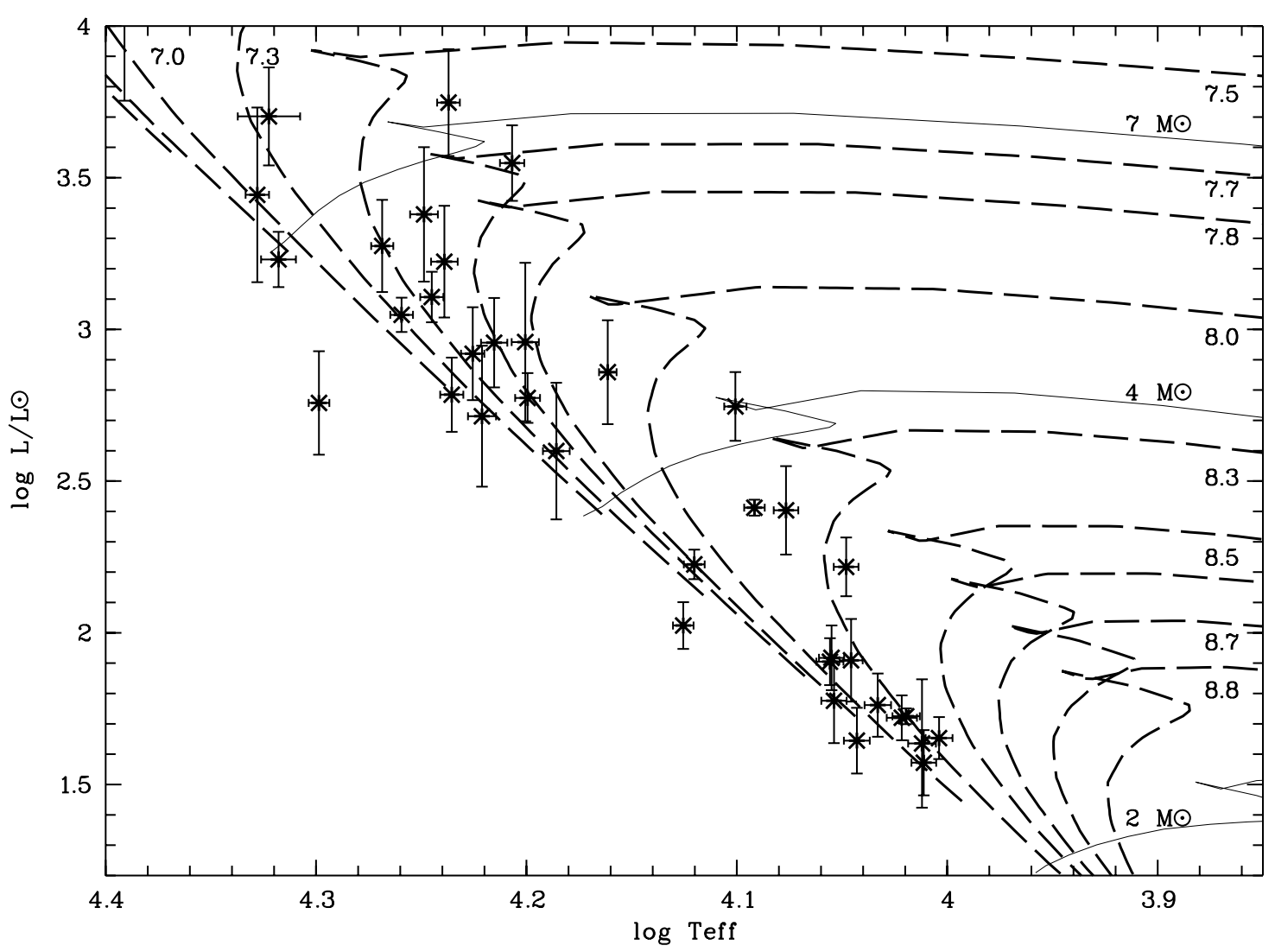

Fig. 4. The $\log T_{\text {eff }}$ versus $\log L / L \odot$ for the primary stars with the evolutionary tracks (thin line) from Schaller et al. (1992) and the isochrones (thick dashed line) from Meynet et al. (1993). For the simplicity of this figure, only 3 evolutionary tracks are plotted and 8 isochrones; the ages (yr) on the isochrones are given in logarithmic scale. The star HD $53755 \mathrm{~A}$ is not plotted, due to its high luminosity, compared to the others. The values of the error bars are from Table 5.

We note that the errors are very similar for both sets of models; they are due to observational errors, mainly due to the parallax inaccuracy.

Two stars lie below the ZAMS, HD 127971 and even more so, HD 180183. Their anomalous position cannot be ascribed to the parallax error only; a spectroscopic study of these objects can clarify the origin of this peculiarity.

HD 113791 and HD 53191 lie slightly below the ZAMS and only the upper limit of their age can be computed.

A last remark concerns the primary stars with a $T_{\text {eff }}$ greater than $15000 \mathrm{~K}$ for which we also computed the age using as $T_{\text {eff }}$ value: $\left(T_{\text {eff }}-500\right) ; 500 \mathrm{~K}$ corresponds to the systematic shift between $T_{\text {eff }}$ derived by using different photometric system (see Sect. 3.3). The values are given in the last two columns of Table 7. We have computed the differences between these determinations of ages and masses for the two $T_{\text {eff }}$, using the Meynet et al. (1993) isochrones and Schaller et al. evolutionary tracks (1992). For the ages and the masses these differences are of the same order or even less than those given in Table 6 , ie within the error bars we have determined.

We have assumed that the early-type stars are in a post-main sequence evolutionary phase; we questioned, now, if some of these stars can be in the pre-main sequence phase. None of these stars has been classified, up to now, as an Herbig Ae/Be object (Thé et al. 1994 catalogue). We also checked through the literature for signs of youth, i.e. emission in hydrogen lines or infrared excess, according to the definition given by Thé et al. (1994). Concerning emission in Balmer lines, only 2 stars among the earlytype primaries have been classified as Be: HD 71510 and HD 137387. In fact while HD 137387 is a classical Be star as already said in Sect. 3, the classification of HD 71510 as Be is less certain. For this star, no emission in $\mathrm{H}_{\alpha}$ has been found by Jaschek et al. (1964) in their survey of Be candidates and the star is classified B3 IV in the Michigan catalogue. The classification as Be appears in the CDS database and in the BSC, but we could not find any paper discussing emissions in the spectrum of this star. We are not aware of any infrared excess among these early-type stars; Ray et al. (1995) consider that the infrared flux detected in some systems is due to the dust surrounding the secondary component. Using the near infrared observations JHKL done by Lindroos (1983), we have investigated the position of the early-type primaries in the color diagram $(H-K)$ versus $(J-H)$ by comparison with that of the main sequence stars which intrinsic colours given by Koornneef (1983). We note that the behaviour of these stars in this diagram is normal, taking into account the errors on the photometric observations, except for HD 90972 and HD 137387. The position of HD 90972 can be explain by the fact that it is an SB2 object and 


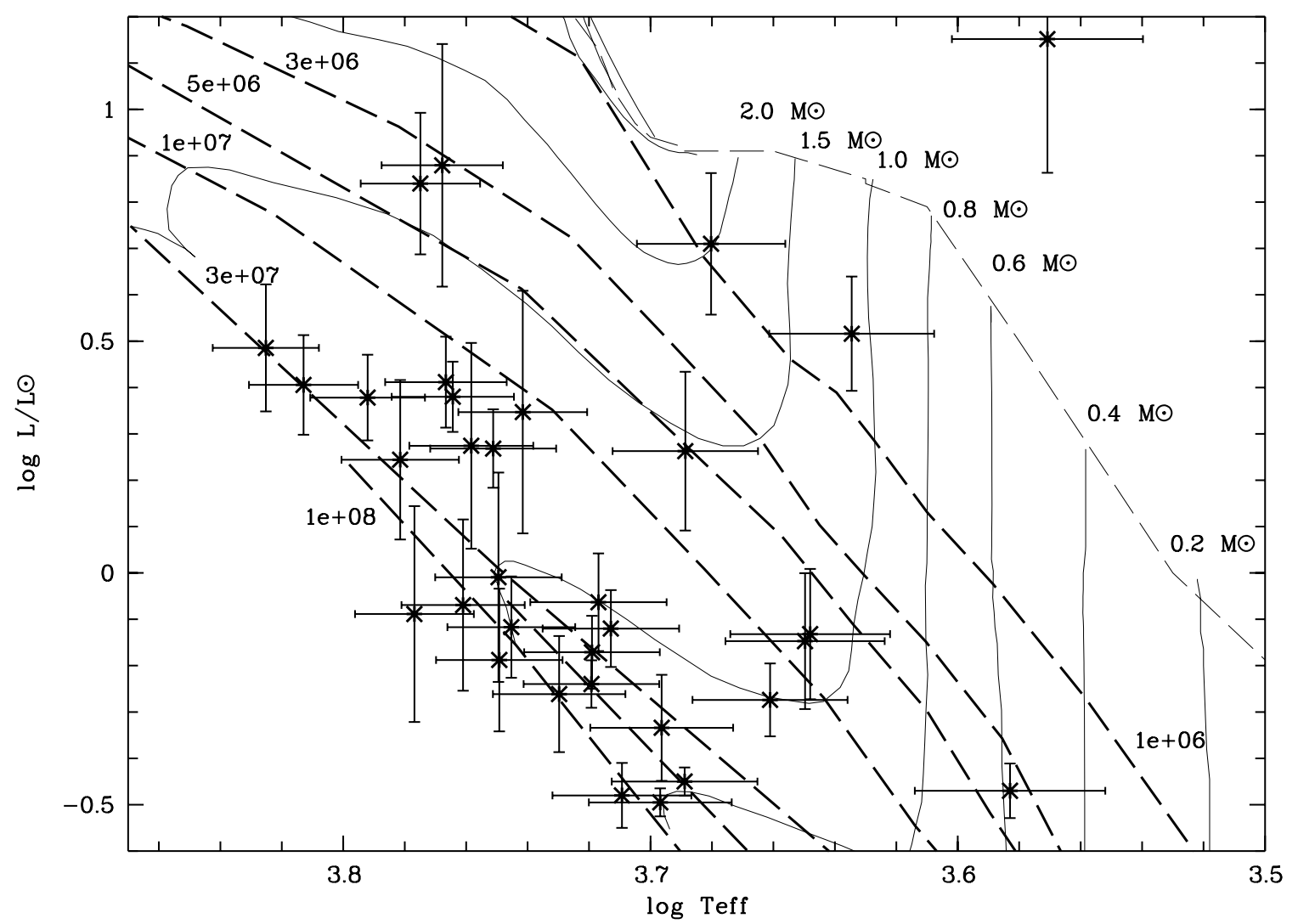

Fig. 5. The $\log T_{\text {eff }}$ versus $\log L / L_{\odot}$ for the secondary stars with the pre-main sequence tracks (thin line) and the isochrones (thick dashed line) computed by Palla \& Stahler (1999). The ages are given in years. The values of the error bars are from Table 5.

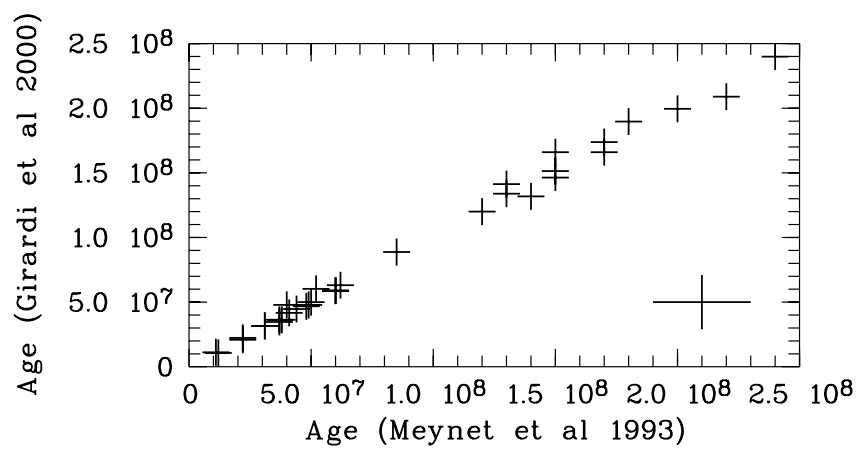

Fig. 6. Comparison of ages for the early-type stars derived from the Meynet et al. (1993) isochrones and those from the Girardi et al. (2000) isochrones. Typical error bars corresponding to the average of the values given Table 6 are plotted in the lower right corner.

that of HD 137387 because it is a Be star. From the analysis by Lada \& Adams (1992) we note that HD 137387 occupies a position in this color diagram coherent with its Be caracter; none of the other early-type primary stars is mixed with any Herbig AeBe object studied by Lada \& Adams (1992). So it is appropriate to use post main sequence evolutionary isochrones to compute the ages and masses of these stars.

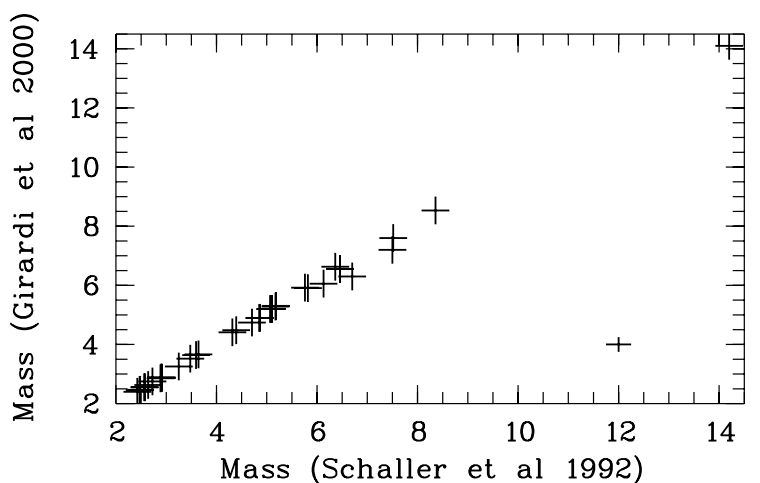

Fig. 7. Comparison of masses for the early-type stars derived from the Meynet et al. (1993) isochrones and those from the Girardi et al. (2000) isochrones. Typical error bars corresponding to the average of the values given Table 6 are plotted in the lower right corner.

\subsection{Ages and masses of the late-type companions}

Pre-main sequence isochrones are used to determine the ages of the late-type companions; as above the uncertainties on the luminosity and on the effective temperature are taken into account.

Recently, several pre-main sequence tracks and isochrones were computed: D'Antona \& Mazzitelli (1998), Baraffe et al. (1998), Palla \& Stahler (1999), Siess et al. (2000). 
Table 6. Ages and masses of the early-type stars computed with the Meynet et al. (1993) isochrones, the Schaller et al. (1992) evolutionary tracks and with the Girardi et al. (2000) evolutionary models. For each parameter the error (in \%) is given.

\begin{tabular}{|c|c|c|c|c|c|c|c|c|c|}
\hline $\mathrm{HD}$ & & Age (yr) & $\%$ & $\mathcal{M} / \mathcal{M} \odot$ & $\%$ & Age (yr) & $\%$ & $\mathcal{M} / \mathcal{M} \odot$ & $\%$ \\
\hline (1) & $(2)$ & $\begin{array}{l}\text { Meynet et al. } \\
\text { (3) }\end{array}$ & $\begin{array}{c}(1993) \\
(4)\end{array}$ & $\begin{array}{l}\text { Schaller et al. } \\
\text { (5) }\end{array}$ & $\begin{array}{c}(1992) \\
(6)\end{array}$ & $\begin{array}{c}\text { Girardi } \\
(7)\end{array}$ & $\begin{array}{l}\text { et } \\
(8)\end{array}$ & $\begin{array}{l}\text { al. } \\
(9)\end{array}$ & $\begin{array}{c}(2000) \\
(10)\end{array}$ \\
\hline 560 & $\mathrm{~A}$ & $1.3 \mathrm{e}+08$ & 33 & 2.9 & 3 & $1.4 \mathrm{e}+08$ & 35 & 2.9 & 5 \\
\hline 33802 & A & $6.2 \mathrm{e}+07$ & 30 & 3.5 & 3 & $6.3 \mathrm{e}+07$ & 29 & 3.5 & 2 \\
\hline 38622 & A & $4.4 \mathrm{e}+07$ & 11 & 6.1 & 4 & $4.5 \mathrm{e}+07$ & 13 & 6.1 & 7 \\
\hline 90972 & A & $2.2 \mathrm{e}+08$ & 5 & 3.2 & 4 & $2.1 \mathrm{e}+08$ & 5 & 3.3 & 4 \\
\hline 108767 & $\mathrm{~A}$ & $2.0 \mathrm{e}+08$ & 18 & 2.6 & 1 & $2.0 \mathrm{e}+08$ & 15 & 2.6 & 1 \\
\hline 113703 & A & $4.8 \mathrm{e}+07$ & 21 & 4.8 & 2 & $4.7 \mathrm{e}+07$ & 21 & 4.9 & 2 \\
\hline 113791 & $\mathrm{~A}$ & - & - & - & - & - & - & - & - \\
\hline 129791 & A & $1.2 \mathrm{e}+08$ & - & 2.4 & 2 & $1.2 \mathrm{e}+08$ & - & 2.4 & 5 \\
\hline 143939 & $\mathrm{~A}$ & $5.0 \mathrm{e}+07$ & - & 2.7 & 3 & $5.0 \mathrm{e}+07$ & - & 2.8 & 3 \\
\hline 17543 & $\mathrm{AB}$ & $8.5 \mathrm{e}+07$ & 5 & 4.7 & 5 & $8.9 e+07$ & 4 & 4.7 & 8 \\
\hline 27638 & $\mathrm{~A}$ & $1.8 \mathrm{e}+08$ & 28 & 2.6 & 3 & $1.9 \mathrm{e}+08$ & 26 & 2.6 & 3 \\
\hline 40494 & A & $5.2 \mathrm{e}+07$ & 16 & 6.7 & 2 & $6.0 \mathrm{e}+07$ & - & 6.3 & - \\
\hline 53191 & A & - & - & - & - & - & - & - & - \\
\hline 60102 & A & $1.5 \mathrm{e}+08$ & - & 2.6 & 6 & $1.5 \mathrm{e}+08$ & 47 & 2.6 & 5 \\
\hline 77484 & A & $1.7 \mathrm{e}+08$ & - & 2.5 & 6 & $1.7 \mathrm{e}+08$ & - & 2.5 & 8 \\
\hline 127304 & A & $2.4 \mathrm{e}+08$ & 19 & 2.5 & 2 & $2.4 \mathrm{e}+08$ & 18 & 2.5 & 5 \\
\hline 8803 & A & $1.7 \mathrm{e}+08$ & 35 & 2.9 & 5 & $1.7 \mathrm{e}+08$ & 31 & 2.9 & 6 \\
\hline 23793 & $\mathrm{AB}$ & $4.9 \mathrm{e}+07$ & 19 & 5.2 & 6 & $4.8 \mathrm{e}+07$ & 22 & 5.3 & 6 \\
\hline 35007 & A & $4.1 \mathrm{e}+07$ & 7 & 6.4 & 10 & $4.2 \mathrm{e}+07$ & 8 & 6.6 & 10 \\
\hline 36013 & $\mathrm{~A}$ & ZAMS & - & 4.9 & 3 & $1.0 \mathrm{e}+07$ & - & 4.9 & 5 \\
\hline 36151 & A & $6.0 \mathrm{e}+07$ & 24 & 5.1 & 11 & $5.9 \mathrm{e}+07$ & 29 & 5.2 & 9 \\
\hline 36779 & $\mathrm{~A}$ & $1.2 \mathrm{e}+07$ & - & 7.5 & 8 & $1.1 \mathrm{e}+07$ & - & 7.6 & 8 \\
\hline 43286 & $\mathrm{~A}$ & $3.7 \mathrm{e}+07$ & - & 4.4 & 6 & $3.5 \mathrm{e}+07$ & - & 4.5 & 6 \\
\hline 63465 & $\mathrm{AB}$ & $4.0 \mathrm{e}+07$ & 24 & 7.5 & 7 & $4.8 \mathrm{e}+07$ & - & 7.2 & - \\
\hline 70309 & $\mathrm{~A}$ & ZAMS & - & 5.1 & 2 & ZAMS & - & 5.2 & 2 \\
\hline 71510 & A & $3.7 \mathrm{e}+07$ & 12 & 5.8 & 5 & $3.6 \mathrm{e}+07$ & 15 & 5.9 & 2 \\
\hline 76566 & AV & $3.8 \mathrm{e}+07$ & 41 & 5.2 & 5 & $3.6 \mathrm{e}+07$ & 46 & 5.3 & 6 \\
\hline 86388 & $\mathrm{~A}$ & $1.4 \mathrm{e}+08$ & 38 & 2.9 & 4 & $1.3 \mathrm{e}+08$ & 30 & 2.9 & 4 \\
\hline 87901 & $\mathrm{~A}$ & $1.5 \mathrm{e}+08$ & 3 & 3.6 & 0 & $1.5 \mathrm{e}+08$ & 5 & 3.7 & 1 \\
\hline 106983 & A & $2.2 \mathrm{e}+07$ & 27 & 5.8 & 3 & $2.1 \mathrm{e}+07$ & 25 & 5.9 & 2 \\
\hline 123445 & A & $1.5 \mathrm{e}+08$ & 13 & 3.6 & 5 & $1.7 \mathrm{e}+08$ & 3 & 3.6 & 5 \\
\hline 127971 & $\mathrm{~A}$ & - & - & - & - & - & - & - & - \\
\hline 137387 & $\mathrm{AV}$ & $2.2 \mathrm{e}+07$ & 14 & 8.4 & 6 & $2.2 \mathrm{e}+07$ & 17 & 8.5 & 6 \\
\hline 138800 & A & $1.3 \mathrm{e}+08$ & 4 & 4.3 & 6 & $1.3 \mathrm{e}+08$ & 5 & 4.4 & 6 \\
\hline 180183 & $\mathrm{~A}$ & - & - & - & - & - & - & - & - \\
\hline 53755 & $\mathrm{ABV}$ & $1.1 \mathrm{e}+07$ & - & 14.2 & - & $1.1 \mathrm{e}+07$ & - & 14.1 & - \\
\hline 174585 & A & $3.1 \mathrm{e}+07$ & 19 & 6.5 & 5 & $3.2 \mathrm{e}+07$ & 22 & 6.6 & 6 \\
\hline
\end{tabular}

These evolutionary models differ for the different initial conditions and for the different physics inputs, in particular the treatment of convection. Since the Baraffe et al. (1998) models are limited to stars with a mass lower than one solar mass, we will not consider these models.

The ages and masses derived for the late-type companions are given in Table 8. We do not discuss in this section the fact that some of these stars can be in a post-main sequence phase; such an hypothesis will be discussed in the last section.

The label "MS" in Table 8 corresponds to a star located on the main sequence or when one of the error bar crosses it. The "*" has the same meaning as in the early type stars section: no computation could be done. 
Table 8. Ages of the late-type stars computed with the isochrones from D'Antona \& Mazzitelli (1998), Palla \& Stahler (1999), Siess et al. (2000), Tout et al. (1999). The masses are computed from the evolutionary models by Palla \& Stahler (1999), Siess et al. (2000), Tout et al. (1999). For these parameters the error (in \%) is given.

\begin{tabular}{|c|c|c|c|c|c|c|c|c|c|c|c|}
\hline $\mathrm{HD}$ & $(2)$ & $\begin{array}{c}\text { Age (yr) } \\
\text { D'Antona } \\
(3)\end{array}$ & $\begin{array}{c}\% \\
\text { et al. } \\
(4)\end{array}$ & $\begin{array}{c}\text { Age (yr) } \\
\text { Palla } \\
(5)\end{array}$ & $\begin{array}{c}\% \\
\text { et al. } \\
(6)\end{array}$ & $\begin{array}{c}\text { Age (yr) } \\
\text { Siess et al. } \\
(7)\end{array}$ & $\begin{array}{c}\text { Age (yr) } \\
\text { Tout et al. } \\
\text { (8) }\end{array}$ & $\begin{array}{c}\mathcal{M} / \mathcal{M} \odot \\
\text { Palla } \\
(9)\end{array}$ & $\begin{array}{c}\% \\
\text { et al. } \\
(10)\end{array}$ & $\begin{array}{c}\mathcal{M} / \mathcal{M} \odot \\
\text { Siess et al. } \\
(11)\end{array}$ & $\begin{array}{c}\mathcal{M} / \mathcal{M} \odot \\
\text { Tout et al. } \\
(12)\end{array}$ \\
\hline 560 & B & $2.0 \mathrm{e}+07$ & 53 & $3.0 \mathrm{e}+07$ & 130 & $3.6 \mathrm{e}+07$ & $3.0 \mathrm{e}+07$ & 0.95 & 5 & 0.91 & 0.98 \\
\hline 33802 & B & $2.5 \mathrm{e}+07$ & 170 & $5.0 \mathrm{e}+07$ & $*$ & $4.7 \mathrm{e}+07$ & $3.7 \mathrm{e}+07$ & 0.90 & 4 & 0.93 & 0.93 \\
\hline 38622 & $\mathrm{C}$ & $3.0 \mathrm{e}+07$ & $*$ & $1.0 \mathrm{e}+08$ & $*$ & $*$ & $1.1 \mathrm{e}+08$ & 1.05 & 6 & $*$ & 1.06 \\
\hline 90972 & B & $1.4 \mathrm{e}+07$ & 36 & $1.8 \mathrm{e}+07$ & 28 & $1.8 \mathrm{e}+07$ & $1.5 \mathrm{e}+07$ & 1.25 & 6 & 1.22 & 1.31 \\
\hline 108767 & B & $4.0 \mathrm{e}+07$ & $*$ & $9.0 \mathrm{e}+07$ & $*$ & $*$ & $9.3 \mathrm{e}+07$ & 0.80 & 0 & $*$ & 0.85 \\
\hline 113703 & B & $1.5 \mathrm{e}+07$ & 57 & $2.5 \mathrm{e}+07$ & 64 & $2.9 \mathrm{e}+07$ & $2.3 \mathrm{e}+07$ & 0.98 & 8 & 0.99 & 1.04 \\
\hline 113791 & B & $1.8 \mathrm{e}+07$ & $*$ & $2.2 \mathrm{e}+07$ & 27 & $1.1 \mathrm{e}+09$ & $2.1 \mathrm{e}+07$ & 1.22 & 5 & 1.28 & 1.25 \\
\hline 129791 & B & $*$ & $*$ & $*$ & $*$ & $*$ & $*$ & $*$ & $*$ & $*$ & $*$ \\
\hline 143939 & B & $2.5 \mathrm{e}+06$ & 74 & $7.0 \mathrm{e}+06$ & 73 & $8.3 e+06$ & $6.0 \mathrm{e}+06$ & 1.15 & 9 & 1.14 & 1.15 \\
\hline 17543 & $\mathrm{C}$ & $2.4 \mathrm{e}+07$ & $*$ & $2.8 \mathrm{e}+07$ & $*$ & $*$ & $2.5 \mathrm{e}+07$ & 1.18 & 4 & $*$ & 1.17 \\
\hline 27638 & B & $1.2 \mathrm{e}+07$ & 38 & $1.5 \mathrm{e}+07$ & 37 & $1.9 \mathrm{e}+07$ & $1.5 \mathrm{e}+07$ & 1.25 & 4 & 1.25 & 1.30 \\
\hline 40494 & B & $3.5 \mathrm{e}+07$ & $*$ & $1.0 \mathrm{e}+08$ & $*$ & $*$ & $7.6 \mathrm{e}+07$ & 0.94 & 4 & $*$ & 0.94 \\
\hline 53191 & B & $3.0 \mathrm{e}+07$ & $*$ & $8.0 \mathrm{e}+07$ & $*$ & $*$ & $3.9 \mathrm{e}+07$ & 1.00 & 3 & $*$ & 0.99 \\
\hline 60102 & B & $1.5 \mathrm{e}+07$ & 53 & $2.5 \mathrm{e}+07$ & 30 & $*$ & $2.0 \mathrm{e}+07$ & 1.00 & 10 & $*$ & 1.08 \\
\hline 77484 & B & $*$ & $*$ & $*$ & $*$ & $*$ & $*$ & $*$ & $*$ & $*$ & $*$ \\
\hline 127304 & B & $5.0 \mathrm{e}+07$ & * & $1.0 \mathrm{e}+08$ & $*$ & * & $1.0 \mathrm{e}+08$ & 0.84 & 4 & $*$ & 0.89 \\
\hline 8803 & B & $*$ & * & $3.0 \mathrm{e}+07$ & * & $*$ & $1.1 \mathrm{e}+08$ & 1.30 & 3 & $*$ & 1.40 \\
\hline 23793 & B & $*$ & $*$ & * & $*$ & $*$ & $*$ & $*$ & $*$ & $*$ & $*$ \\
\hline 35007 & $\mathrm{C}$ & $1.5 \mathrm{e}+07$ & 50 & $2.0 \mathrm{e}+07$ & 50 & $2.2 \mathrm{e}+07$ & $1.7 \mathrm{e}+07$ & 1.18 & 8 & 1.12 & 1.23 \\
\hline 36013 & B & $*$ & $*$ & $*$ & $*$ & $*$ & $1.1 \mathrm{e}+08$ & $*$ & $*$ & $*$ & 1.13 \\
\hline 36151 & B & $5.0 \mathrm{e}+06$ & 65 & $3.0 \mathrm{e}+06$ & 95 & $7.6 \mathrm{e}+06$ & $5.4 \mathrm{e}+06$ & 1.75 & 20 & 1.75 & 1.89 \\
\hline 36151 & $\mathrm{X}$ & $8.5 \mathrm{e}+06$ & 71 & $1.3 e+07$ & 77 & $1.5 \mathrm{e}+07$ & $9.9 \mathrm{e}+06$ & 1.28 & 16 & 1.30 & 1.43 \\
\hline 36779 & B & $*$ & $*$ & $*$ & $*$ & $*$ & $5.0 \mathrm{e}+03$ & $*$ & $*$ & $*$ & 0.38 \\
\hline 43286 & B & $2.5 \mathrm{e}+07$ & $*$ & $4.0 \mathrm{e}+07$ & $*$ & $4.5 \mathrm{e}+07$ & $3.0 \mathrm{e}+07$ & 1.00 & 7 & 0.32 & 1.03 \\
\hline 63465 & B & $*$ & $*$ & $*$ & $*$ & $*$ & $*$ & $*$ & $*$ & $*$ & $*$ \\
\hline 70309 & B & $2.0 \mathrm{e}+05$ & 58 & $\leq 1.0 \mathrm{e}+06$ & 0 & $7.4 \mathrm{e}+05$ & $4.3 \mathrm{e}+05$ & 1.10 & 36 & 1.07 & 0.82 \\
\hline 71510 & $\mathrm{C}$ & $1.3 \mathrm{e}+07$ & 38 & $1.8 \mathrm{e}+07$ & 33 & $2.1 \mathrm{e}+07$ & $1.5 \mathrm{e}+07$ & 1.18 & 7 & 1.17 & 1.26 \\
\hline 76566 & B & $5.0 \mathrm{e}+07$ & $*$ & MS & $*$ & * & $1.1 \mathrm{e}+08$ & 0.98 & 4 & $*$ & 1.01 \\
\hline 86388 & B & $*$ & $*$ & $3.0 \mathrm{e}+07$ & $*$ & $*$ & $1.1 \mathrm{e}+08$ & 1.28 & 2 & $*$ & 1.33 \\
\hline 87901 & B & $3.0 \mathrm{e}+07$ & 58 & $5.0 \mathrm{e}+07$ & 70 & $5.2 \mathrm{e}+07$ & $4.5 \mathrm{e}+07$ & 0.82 & 3 & 0.81 & 0.84 \\
\hline 106983 & B & $2.2 \mathrm{e}+06$ & 91 & $3.8 \mathrm{e}+06$ & 99 & $4.0 \mathrm{e}+06$ & $3.4 \mathrm{e}+06$ & 0.59 & 38 & 0.56 & 0.58 \\
\hline 123445 & B & $3.0 \mathrm{e}+06$ & 75 & $8.0 \mathrm{e}+06$ & 69 & $*$ & $6.7 \mathrm{e}+06$ & 1.15 & 15 & $*$ & 1.15 \\
\hline 127971 & B & $7.0 \mathrm{e}+06$ & 71 & $1.8 \mathrm{e}+07$ & 46 & $1.8 \mathrm{e}+07$ & $1.4 \mathrm{e}+07$ & 1.00 & 8 & 1.03 & 1.05 \\
\hline 137387 & B & $*$ & $*$ & $*$ & $*$ & $*$ & $*$ & $*$ & $*$ & $*$ & $*$ \\
\hline 138800 & $\mathrm{X}$ & $2.0 \mathrm{e}+07$ & 75 & $3.8 \mathrm{e}+07$ & 99 & $4.0 \mathrm{e}+07$ & $3.4 \mathrm{e}+07$ & 0.88 & 7 & 0.68 & 0.91 \\
\hline 180183 & B & $2.2 \mathrm{e}+06$ & 84 & $5.0 \mathrm{e}+06$ & 70 & $6.4 \mathrm{e}+06$ & $3.7 \mathrm{e}+06$ & 1.48 & 17 & 1.52 & 1.51 \\
\hline 53755 & B & $*$ & $*$ & $*$ & $*$ & $*$ & $*$ & $*$ & $*$ & $*$ & * \\
\hline 174585 & B & $4.6 \mathrm{e}+05$ & 87 & $1.0 \mathrm{e}+06$ & 50 & $*$ & $6.9 \mathrm{e}+05$ & 2.00 & 25 & $*$ & 1.48 \\
\hline 174585 & $\mathrm{C}$ & $7.0 \mathrm{e}+06$ & 36 & $4.0 \mathrm{e}+06$ & 53 & $9.0 \mathrm{e}+06$ & $7.1 \mathrm{e}+05$ & 1.70 & 13 & 1.65 & 1.75 \\
\hline
\end{tabular}

Discussion on the position occupied by individual target in the HR diagram will be given in the next section.

As for the early type stars, the errors on $T_{\text {eff }}$ and $\log g$ have been added and subtracted (error bars in Fig. 5) to compute the error box on age and mass for each star, for the D'Antona \& Mazitelli (1998) isochrones and those by Palla \& Stahler (1999). The extreme values of those parameters are given Table 9 , for each star.

In Table 8 the ages computed from the evolutionary models mentioned above are given in Cols. 3, 5 and 7 . The values from Tout et al. (1999) (Col. 8) will be discussed in the next section. For the ages computed from the D'Antonna \& Mazitelli and Palla \& Stahler models, the errors are also given, expressed in percentage (Cols. 4 and 6$)$.

The masses (in Cols. 9 and 11) have been computed with the Palla \& Stahler (1999) and the Siess et al. (2000) tracks; the error in percentage (Col. 10) is given for the Palla \& Stahler masses. The masses computed from Tout et al. (1999) (Col. 12) will be discussed later.

For a given $T_{\text {eff }}$ and $\log L / L_{\odot}$ the D'Antona \& Mazzitelli (1998) isochrones present a considerable difference with the other sources. These large differences are analysed by both Palla \& Stahler (1999) and 


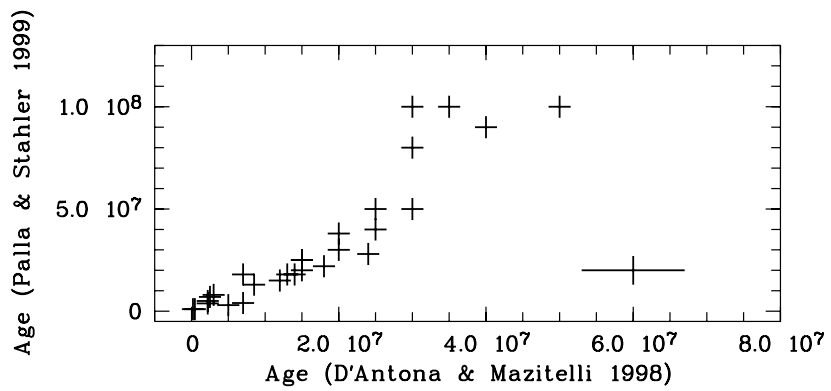

Fig. 8. Comparison between the ages computed from D'Antona \& Mazitelli (1998) isochrones and those from Palla \& Stahler (1999) isochrones. Typical error bars corresponding to the average of the values given Table 8 are plotted in the lower right corner.

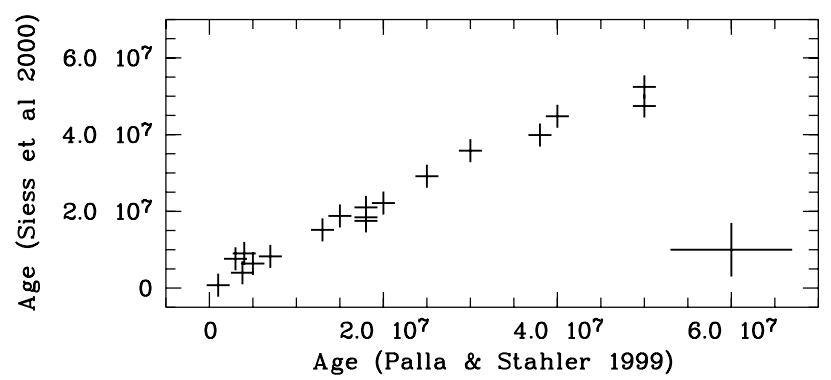

Fig. 9. Comparison between the ages computed from Palla \& Stahler (1999) isochrones and those from Siess et al. (2000) isochrones. The star HD 113791 B is not plotted due to its extreme age computed from the Siess et al. isochrones. Typical error bars are plotted in the lower right corner.

Siess et al. (2000) and will not be examined again here. Figure 8 compares the ages computed by using D'Antona \& Mazzitelli (1998) isochrones to those from the Palla \& Stahler (1999) isochrones; these large differences reflect the behaviour of these isochrones which are very different in the domain of interest for the targets analysed in this paper.

The comparison of the ages obtained from the isochrones by Palla \& Stahler (1999) to those by Siess et al. (2000) shows a systematic difference of about $10 \%$, Siess et al. predicting higher ages (Fig. 9).

The masses derived from Palla \& Stahler (1999) evolutionary tracks and those from Siess et al. (2000) tracks are comparable (Fig. 10).

\section{Ages analysis}

The ages of primaries and secondaries obtained in previous sections are compared in order to check if they are compatible with the hypothesis of physical systems. In Fig. 11 the ages of both components are plotted with their error bars. The ages retained in this figure are those from Meynet et al. (1993) and Palla \& Stahler (1999) isochrones. Formally, we should add the time elapsed during the pre-main sequence phase to the ages of the primaries. For stars in this range of masses this time is less than $3 \times 10^{6}$ years (Palla \& Stahler 1999) and so it has a negligible influence on the total stellar age.

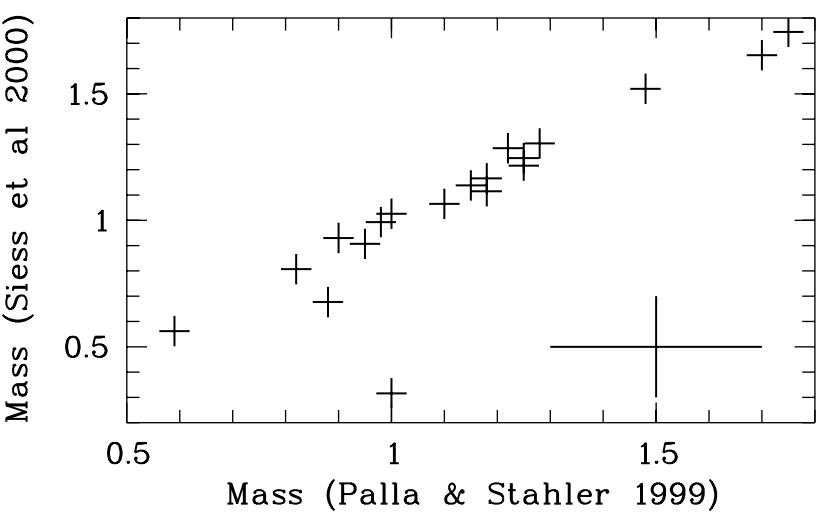

Fig. 10. Comparison between the masses, expressed in solar unit, computed from Palla \& Stahler (1999) isochrones and those from Siess et al. (2000) isochrones. The star HD 43286 B has discrepant values between these two sets of tracks. Typical error bars are plotted in the lower right corner.

For the systems HD 127971 and 180183, the position of the A component in the HR diagram far below the ZAMS prevents any age determination. For some systems the age has not been computed for the late-type component due to the lack of $T_{\text {eff }}$ value and so the physical association to its primary cannot be discussed; these systems are: HD 129791, HD 77484, HD 23793, HD 63465, and HD 137387.

We continue to retain in the following the separation in the three groups defined spectroscopically by PPR, to which we have added as a fourth group the stars analysed by MMR only.

\subsection{Stars of the first PPR group}

For the systems: HD 560, HD 33802, HD 38622, HD 113703, HD 143939 the ages are compatible with a physical association between the two components, due to the overlap of the age error bars. For HD 113791, the A component is so close to the ZAMS, that we may admit the physical association between the two components. The radial velocities of the two components are similar according to MMR for the first 3 stars, and Duflot et al. (1995) for HD 113703 and HD 113791; no measure of the radial velocities is available for HD 143939. The positions of HD $38622 \mathrm{~A}$ and C in Fig. 11 are inverted: we note that for the system HD 38622 the age of the late type component is higher than that of its primary, i.e. ages are incompatible with the mass values; as we already mentioned, the A component cannot be in a pre-main sequence phase due to the lack of a spectral signature of youth.

For HD 90972 the ages seem incompatible with the hypothesis of a physical system; we recall that the primary of this system is an SB2 and so the computed luminosity of HD 90972A is too high, but a duplicity effect, even by a factor of 2 on the luminosity, is not sufficient to explain the large age difference from its visual $\mathrm{B}$ component.

HD 108767: the age of the secondary is considerably lower than that of the primary, and on the basis of ages 


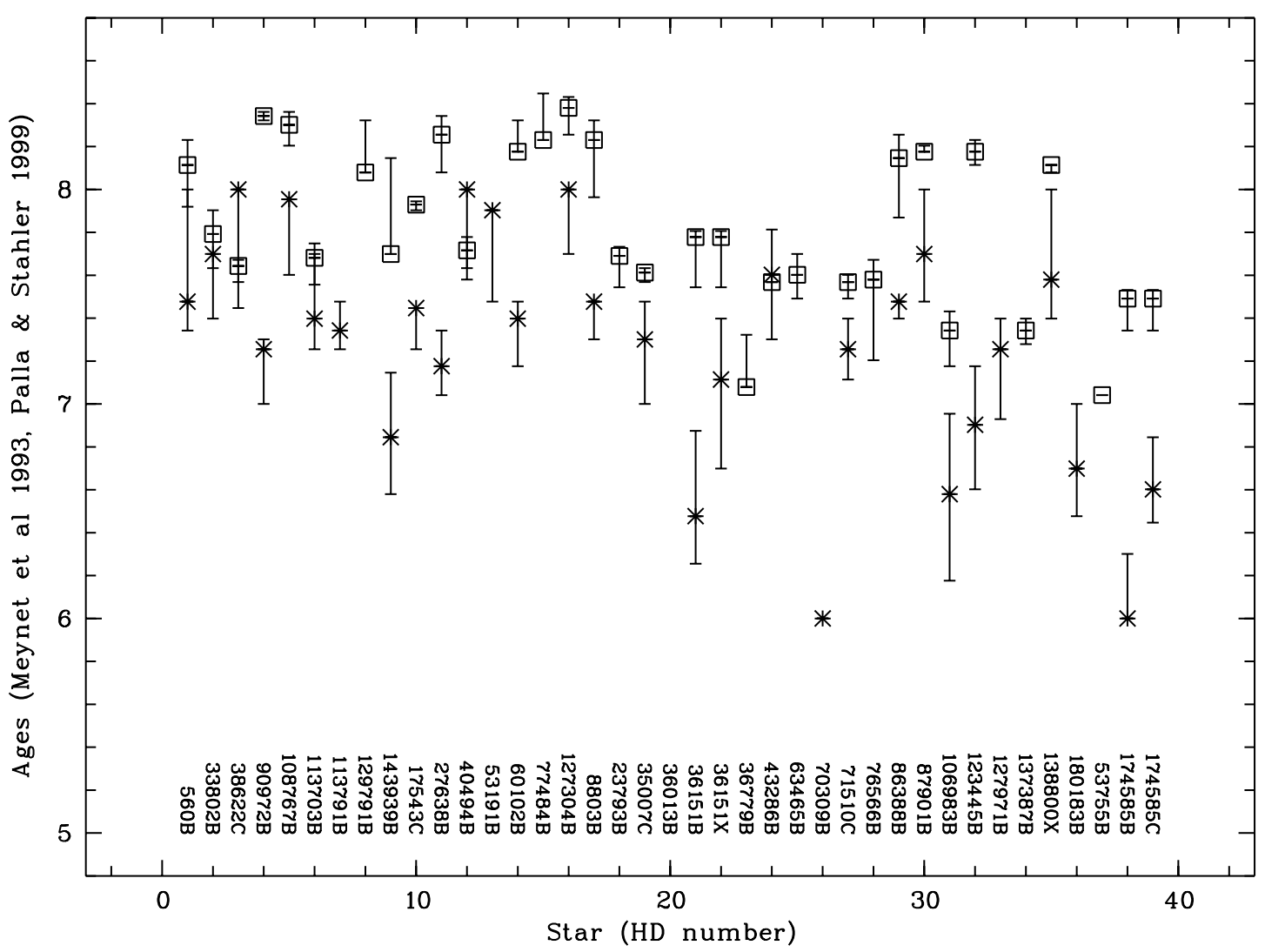

Fig. 11. Comparison between the ages of the early-type stars (square) and the ages of the late-type stars (*), expressed in logarithm of year, computed respectively from the Meynet et al. (1993) isochrones, and the Palla \& Stahler (1999) isochrones. The values of the error bars for the early and late-type stars are respectively from Tables 7 and 9 . Only stars with age values are plotted; the indication of the secondary is explicitly given. The stars from the first PPR group are from number 1 to 9 , those from the second PPR group: number 10 to 16, for the third PPR group number 17 to 36 and the three last one are those from MMR only.

the components should not be physically associated, moreover the ages are incompatible with the mass values. We note that MMR found similar radial velocities for the two components.

We remark that among the stars of this group, HD $143939 \mathrm{~B}$ has the lowest age $\left(7.0 \times 10^{6} \mathrm{yr}\right)$ according to our estimation and has also strong characteristics of a pre-main sequence star, according to $\mathrm{PPR}$, in particular an emission on the $\mathrm{H}_{\alpha}$ as well as a strong CaII $\mathrm{K}$ emission.

For all these stars, the X-ray emissions discussed by Huélamo et al. (2000) (hereafter noted HNSSZ) reinforce the hypothesis of the youth of the secondaries.

\subsection{Stars of second PPR group}

HD 17543, HD 40494, HD 53191: the late-type companions of these 3 systems are close to the main sequence, so, by taking into account the uncertainties on their parameters, their ages are compatible with those determined for the early type stars. There is another system: HD 40494 A and B whose ages are inverted; no interpretation can be found except that of a mass transfer, but the distance between $\mathrm{A}$ and $\mathrm{B}$ stars is too high to make this mechanism efficient. It is noted by HNSSZ that HD 40494B has also a strong Li 6707 feature but no X-rays emission, sign of youth.

HD 27638: the late-type companion HD $27638 \mathrm{~B}$ is an SB2 (PPR, MMR) with rather similar components according to these authors. The luminosity computed for HD $27638 \mathrm{~B}$ is affected by this binarity and even if we take this effect into account, by lowering the luminosity of the $\mathrm{B}$ component by a factor 2 , the binarity effect it is not sufficient to reconcile the two ages. MMR suggested that the two late-type components could be on the ZAMS, instead to be in a pre-main sequence phase. We tested this hypothesis by plotting this star in an HR diagram with post-main sequence isochrones. If we lower its luminosity by a factor of 2 , the B component is located on the ZAMS, and according to its mass, its age is compatible with that of the A component; in such a framework, it will remain to reconcile the strong intensity of the Li 6707 with such an evolutionary phase.

HD 60102 and HD 127304 cannot be considered as physical systems on the basis of the ages of their components. 
For the two systems, HD 27638 and HD 127304, MMR found coherent radial velocities values, compatible with a physical association: the radial velocity has not yet been measured for HD 60102. However we recall that HNSSZ having found clear evidence of youth in HD 60102B, infer that this star has all the characteristics to be considered bound to its primary. An investigation of the primary is necessary to elucidate the discrepant ages we have obtained.

\subsection{Stars of the third PPR group}

At first glance from Fig. 11 we note that very few systems may be considered as physical on the basis of similar age for the two components. They are: HD 43286, HD 76566 and also HD 36013 if we consider that even being below the MS the error bars reach it. MMR excluded that HD 43286 A and B are physically associated on the basis of their discrepant radial velocities.

According to Duflot et al. (1995), the two components of the systems HD $\mathbf{8 8 0 3}$ and HD $\mathbf{8 7 9 0 1}$ have the same radial velocity value but the ages are discordant.

The star HD $\mathbf{8 6 3 8 8} \mathbf{B}$ is almost on the MS, nevertheless the age of the two components seem too different to compose a physical system. The primary variability of unknown type (U) has been discovered from Hipparcos data.

We note that the position of the star HD 36779 B, in the HR diagram, above the birth-line is such that the system is not physical; the radial velocities measured by MMR for the two stars A and B reinforce this conclusion.

In the HR diagram with pre-main sequence tracks (Fig. 5) some stars, of this group, lie above the MS: HD 35007 C, HD 36151 B, HD $36151 \mathrm{X}$, HD 70309 B, HD 71510 C, HD 106983 B and HD 123445 B. Their ages are not compatible with those of their early type companion; we remind that for HD 36151 A, B and HD 35007 A, C the radial velocities measured by MMR are not similar. Moreover, according to PPR, the spectra of these stars do not present any of the spectroscopic signatures characteristic of young objects, such as those detected for the stars in the PPR group I. These stars cannot alternatively be considered in a post ZAMS phase, being too far above it, so too evolved, to be associated with the early type ones. We conclude that the hypothesis that the two components have the same distance is not satisfied. The same conclusion is obtained for HD $138800 \mathrm{X}$ even if that one is closer to the MS.

\subsection{Stars in fourth group, MMR}

For HD 53755 the very large error on the parallax determination prevents any discussion of this system. We can simply note that the late type star cannot be considered in a pre-main sequence phase, being far above the birthline; the same conclusion was reached by MMR. We have already noted in Sect. 2 some of the peculiar properties of this system.

If $\mathrm{HD} \mathbf{1 7 4 5 8 5} \mathrm{B}$ and $\mathrm{C}$ are in pre-main sequence phases, they would be very young and should show strong spectroscopic signatures of their youth, but these signatures are not observed. If we consider them in a postmain sequence phase, the stars would be too old (more than $10^{9}$ years) to be associated with the not yet evolved HD $174585 \mathrm{~A}$. The discrepant radial velocity values measured by MMR are also in favour of the optical system interpretation.

\section{Discussion}

The comparison of the ages of early- and late-type components of a visual binary system allows us to select the likely physical systems independent of any assumption except that of a common distance of the system components.

The interest of such a method is that it permits, in principle, to detect physical systems even if the late-type components have lost the strong spectroscopic signatures of youth, being already too close to the MS. This achievement, if possible, would give access to the full sequence of stars in the latest phase of their pTT life.

The previous section has demonstrated that the errors on $T_{\text {eff }}$ and even more, those on the luminosity, are such that it is easier to exclude the systems not physically bound rather than to put some strong constraint on the physical ones.

The accuracy of the observational and theoretical data is not sufficient to assign stringent values to the age for several of the examined systems. However, in spite of the large error bars, we have established that we could select a number of systems which, according to their position in the HR diagram, may be physically associated. This represents one of the information sources to select true pTT among the secondaries of the systems already studied by PPR, MMR and HNSSZ.

Most of the inconsistent ages have been found for systems belonging to the PPR third group, so confirming the validity of the spectroscopic selection, but this approach adds the possibility of detecting systems with a late component that has already lost the last spectroscopic signature of the pTT phase, the presence of the Li I 6707 feature, being already at the end of this evolutionary phase.

We note also that we have rejected, on the similarity of the ages, some systems having coherent radial velocities between the two components, but we do not give too much weight to this comparison for the following reason. The radial velocity analysis by MMR is based on the values available from the literature (Abt \& Biggs 1972) for the early-type primaries and on measured velocities on their own spectra for the late type secondaries. Concerning the primaries, the quoted catalogue does not provide any mean value and we have no information on how MMR selected the values as well as the resulting error; it can be seen that, for some stars, the differences between the various radial velocity values are larger than the error box 
used for the selection of coincident radial velocity values, by MMR, that is $3 \mathrm{~km} \mathrm{~s}^{-1}$. We do not know either the accuracy of MMR radial velocity measurements and how these radial velocities have been compared to other velocities obtained with different spectrographs. Detailed radial velocity measurements for all these stars are clearly needed.

Table 10 presents a summary of these results and of those obtained by PPR and MMR. We have added in this table the results obtained by HNSSZ from their study of the X-ray emission of the Lindroos binary systems, an $\mathrm{X}$-ray emission being considered a sign of youth. There are 6 systems (HD 90972, HD 108767, HD 60102, HD 127304, HD 86388 and HD 87901) for which the stars cannot be considered as physically bounded on the basis of their ages, but with X-ray emission from the late-type component. We conclude that the late-type star is a young object not related physically to the primary early-type. There is also the inverse result, i.e. systems physically bounded according to their ages but with no X-ray emission detected from the late-type companion: HD 27638 and HD 40494. We note that even if these stars are "young" according to their computed age, in the HR diagram these stars are close to the main sequence, so they may have lost any X-ray emission, but on the other hand they still show $\mathrm{Li}$ absorption. It is beyond the scope of this paper to discuss any relation between the ages so determined and the intensity of the X-ray emission, for the late-type companion, but such a comparison would be worthwhile to have a better understanding of these systems and should be expanded, when possible, to a larger sample.

Figure 11 shows that the ages we derived for the primaries are systematically higher than those of the secondaries, independent of the groups to which they belong, except for HD 33802 and HD 40494. The difference between the ages, even when it is within the error bars, is almost always in one direction. This same effect has already been noticed by Lindroos (1986), who studied these systems with a similar approach. At that time only groundbased parallaxes and less advanced evolutionary models and isochrones were available. Lindroos discussed in detail the possible origin of this discrepancy and suggested, as a possible explanation, that the ages of the Iben models may be underestimated because the contracting models do not include the fast rotation which is expected in real pre-main sequence stars; by including the rotation the contraction time should become longer, by as much as twice, for low-mass stars (see Sect. 4 of this quoted paper).

One additional physical effect has been considered recently by Tout et al. (1999): the accretion mechanism during the pre-main sequence phase.

The ages and masses of the late-type components have been computed by Tout (private communication), and are given Table 8 (Cols. 8 and 12); Fig. 12 displays the comparison between these ages and those determined from the Palla \& Stahler isochrones. In this figure some stars lie outside the mean relation: HD 8803, HD 40494, HD 53191 and HD 86388. For HD 8803 and HD 86388 we can explain

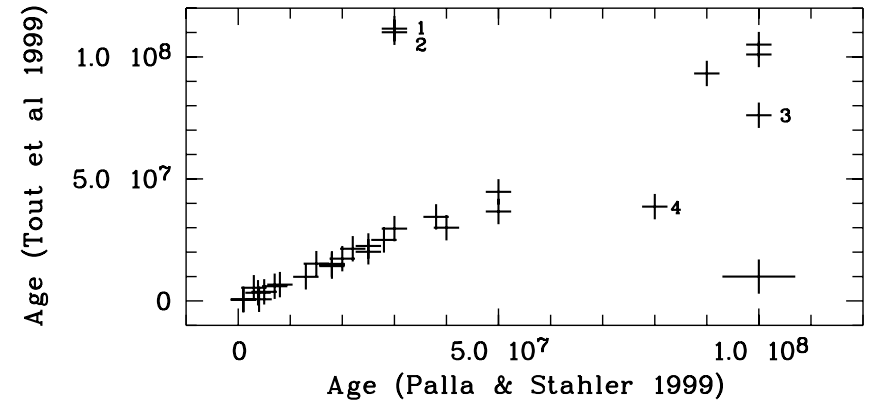

Fig. 12. Comparison between the ages of the late-type stars computed from the Palla \& Stahler (1999) isochrones and the Tout et al. (1999) isochrones. $(1=$ HD 8803B; $2=$ HD 86388B; $3=$ HD 40494B; $4=$ HD 53191B). Typical error bars are plotted in the lower right corner.

the difference according to the analysis by Tout et al. (1999); their $T_{\text {eff }}$ is such that the accreting models make these stars older. If we accept the ages given by the Tout et al. (1999) isochrones, the stars in the systems HD 8803 and HD 86388 are physically linked. For the two other systems HD 40494 and HD 53191 we do not have any explanation since they appear younger according to the Tout et al. (1999) isochrones; their $T_{\text {eff }} \mathrm{s}$ are similar to those of several other stars, in particular to HD 127304 which has the same age from Tout et al. (1999) and Palla \& Stahler (1999) isochrones.

We notice that among the systems of our sample, the largest differences, in age, refer to HD 90972 and HD 27638. The primary of HD 90972 is known to be an SB2 star and so the luminosity we have computed for HD 90972A is certainly too high. The same applies to HD 27638, for which the B component is an SB2 too. We may speculate that these are not the single exceptions and that probably other early-type stars are not yet detected close binaries.

Some of the stars (HD 560, HD 108767, HD 127304, HD 71510, HD 87901, HD 53755 and HD 174585) have been observed by speckle interferometry by the CHARA group and all with negative result, so that a possible companion must have an angular separation below 0.03 arcsec, the resolution of these speckle observations and could be detectabled by spectral analysis. We remark that only the spectra of the secondaries have been analysed up to now, while no study of the spectra of the primaries have been done.

An indication of the existence of undetected companions of the A components of these systems is given by the detection of X-ray emission from some of these earlytype stars (HZSSN) which is difficult to explain if the star is single. The hypothesis of an undetected companion responsible for the $\mathrm{X}$-ray emission requires that this is a late-type star, so its luminosity should not seriously affect the age value we have derived; the companion which is required to explain a too-high primary luminosity must be more luminous, so we can suspect that some primaries are in fact clumps of more than two stars. 
Table 10. Physical and optical systems according to this study, PPR and MMR. In the column labelled X-rays, the results of X-ray analysis by HNSSZ are given; the symbols +, - and ? represent respectively and X-ray emission, no emission and not analysed target. The distance has been computed for each target from the parallax as well as the corresponding projected separation in AU between the components. The error (in \%) on the projected separation, given in the last column, has been computed using the error on the parallax for the distance and taking an error of 0.1 arcsec for the determination of the angular separation of the stars.

\begin{tabular}{|c|c|c|c|c|c|c|c|c|}
\hline $\mathrm{HD}$ & & this study & MMR & X-rays & Dist (pc) & $\operatorname{Sep}\left({ }^{\prime \prime}\right)$ & Sep (AU) & $\%$ \\
\hline \multicolumn{9}{|l|}{ I } \\
\hline 560 & $A+B$ & yes & yes pTTS & + & 100 & 7.7 & 767 & 10 \\
\hline 33802 & $A+B$ & yes & yes pTTS & + & 74 & 12.7 & 938 & 6 \\
\hline 38622 & $\mathrm{~A}+\mathrm{C}$ & yes & yes pTTS & + & 245 & 24.9 & 6100 & 21 \\
\hline 90972 & $\mathrm{~A}+\mathrm{B}$ & no & & + & 147 & 11.0 & 1622 & 12 \\
\hline 108767 & $\mathrm{~A}+\mathrm{B}$ & no & yes pTTS & + & 27 & 24.2 & 652 & 2 \\
\hline 113703 & $A+B$ & yes & & + & 127 & 11.4 & 1446 & 10 \\
\hline 113791 & $A+B$ & yes & & + & 126 & 25.1 & 3168 & 11 \\
\hline 129791 & $\mathrm{~A}+\mathrm{B}$ & - & & + & 130 & 35.3 & 4570 & 12 \\
\hline 143939 & $\mathrm{~A}+\mathrm{B}$ & yes & & + & 168 & 8.6 & 1440 & 17 \\
\hline \multicolumn{9}{|l|}{ II } \\
\hline 17543 & $\mathrm{~A}+\mathrm{C}$ & yes & & + & 185 & 25.2 & 4656 & 20 \\
\hline 27638 & $A+B$ & yes & yes (ZAMS) & - & 82 & 19.4 & 1591 & 9 \\
\hline 40494 & $A+B$ & yes & & - & 262 & 33.8 & 8844 & 14 \\
\hline 53191 & $A+B$ & yes & & - & 207 & 17.0 & 3525 & 13 \\
\hline 60102 & $\mathrm{~A}+\mathrm{B}$ & no & & + & 207 & 16.4 & 3387 & 12 \\
\hline 77484 & $\mathrm{~A}+\mathrm{B}$ & - & & $?$ & 250 & 4.4 & 1099 & 26 \\
\hline 127304 & $\mathrm{~A}+\mathrm{B}$ & no & yes pTTS & + & 107 & 25.8 & 2758 & 8 \\
\hline \multicolumn{9}{|l|}{ III } \\
\hline 8803 & $A+B$ & no & & $?$ & 160 & 6.0 & 960 & 17 \\
\hline 23793 & $\mathrm{~A}+\mathrm{B}$ & - & & $?$ & 173 & 9.0 & 1559 & 18 \\
\hline 35007 & $\mathrm{~A}+\mathrm{C}$ & no & no (VR) MS & $?$ & 330 & 37.6 & 12403 & 26 \\
\hline 36013 & $\mathrm{~A}+\mathrm{B}$ & yes & & $?$ & 303 & 25.0 & 7572 & 27 \\
\hline 36151 & $\mathrm{~A}+\mathrm{B}$ & no & & $?$ & 370 & 48.6 & 17991 & 30 \\
\hline 36151 & $\mathrm{~A}+\mathrm{X}$ & no & no (VR) MS & $?$ & 370 & 45.0 & 16658 & 30 \\
\hline 36779 & $\mathrm{~A}+\mathrm{B}$ & no & yes (VR) but evolved & $?$ & 380 & 27.5 & 10451 & 33 \\
\hline 43286 & $\mathrm{~A}+\mathrm{B}$ & yes & no (VR) MS & $?$ & 308 & 18.3 & 5628 & 26 \\
\hline 63465 & $\mathrm{~A}+\mathrm{B}$ & - & & $?$ & 380 & 10.9 & 4142 & 21 \\
\hline 70309 & $\mathrm{~A}+\mathrm{B}$ & no & & $?$ & 253 & 42.5 & 10727 & 14 \\
\hline 71510 & $\mathrm{~A}+\mathrm{C}$ & no & & $?$ & 207 & 34.9 & 7207 & 10 \\
\hline 76566 & $\mathrm{~A}+\mathrm{B}$ & yes & & $?$ & 287 & 35.0 & 10024 & 18 \\
\hline 86388 & $\mathrm{~A}+\mathrm{B}$ & no & & + & 177 & 9.2 & 1627 & 13 \\
\hline 87901 & $\mathrm{~A}+\mathrm{B}$ & no & yes (VR) ZAMS & + & 24 & 176.9 & 4201 & 2 \\
\hline 106983 & $\mathrm{~A}+\mathrm{B}$ & no & & $?$ & 111 & 33.8 & 3741 & 6 \\
\hline 112244 & $\mathrm{~A}+\mathrm{B}$ & - & & & & & & \\
\hline 123445 & $\mathrm{~A}+\mathrm{B}$ & no & & - & 219 & 28.6 & 6255 & 17 \\
\hline 127971 & $\mathrm{~A}+\mathrm{B}$ & - & & - & 110 & 26.9 & 2955 & 9 \\
\hline 137387 & $\mathrm{~A}+\mathrm{B}$ & - & & $?$ & 312 & 27.0 & 8433 & 19 \\
\hline 138800 & $\mathrm{~A}+\mathrm{X}$ & no & & $?$ & 225 & 34.0 & 7654 & 13 \\
\hline 162082 & $\mathrm{~A}+\mathrm{B}$ & - & & & & & & \\
\hline 180183 & $\mathrm{~A}+\mathrm{B}$ & - & & $?$ & 244 & 19.4 & 4729 & 20 \\
\hline \multicolumn{9}{|l|}{ IV } \\
\hline 53755 & $\mathrm{~A}+\mathrm{B}$ & - & yes (VR) evolved & $?$ & 1087 & 6.6 & 7170 & 97 \\
\hline 174585 & $\mathrm{~A}+\mathrm{B}$ & no & no (VR) post MS & $?$ & 310 & 35.0 & 10830 & 17 \\
\hline 174585 & $\mathrm{~A}+\mathrm{C}$ & no & no (VR) MS & $?$ & 310 & 58.7 & 18164 & 17 \\
\hline
\end{tabular}

A last point may be considered here: the reality - or not - of physical systems among this list of targets using their geometrical properties. By knowing the distances of these stars we have computed the projected separation between the components expressed in $\mathrm{AU}$; these values are given Table 10 .

In Fig. 13 are plotted the projected separations as a function of the $T_{\text {eff }}$ of the early-type star. We compared 


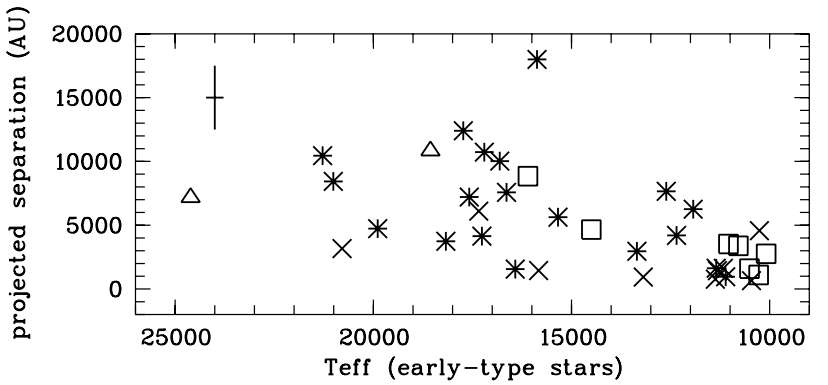

Fig. 13. The projected separation in AU versus the $T_{\text {eff }}$ of the early type star. The different symbols correspond to the PPR groups: $(x)$ for the first group, square for the second group, $\left(^{*}\right)$ for the third group and triangle for the MMR stars. Typical error bars are plotted in the upper left corner. The typical error plotted for the projected separation is for the stars with a projected separation larger than 5000 UA.

this plot to that given by Abt (1988) for the catalogued wide binaries. The trend in Fig. 13 is rather similar to Abt's Fig. 1, specially if we consider only the stars of the first two groups of PPR, so from Fig. 13 we cannot add a new criterion to exclude not physically bounded systems.

If we compare the masses that we have derived for the late-type stars (Table 8) to the masses of the visual binary components listed by Abt et al. (1990) (see Table 5 in this paper) for a totally different sample, but with primaries in the same $T_{\text {eff }}$ range, we note a large similarity between the masses of the stars of the sample analysed here and those of that paper.

A plot of the projected separations according to the groups defined by PPR is given Fig. 14; from this figure we notice that the stars belonging to the third group of PPR, that is the likely optical systems have larger projected separations, compared to the other groups. From this figure we note that there is no strong relation between the physically bounded systems and the values of the projected separation between the components but a possible trend. More data, especially radial velocity measurements, are needed for the late type stars of all the systems to further discuss this point.

As compared to the analysis by Lindroos, the new evolutionary models and refined distances give more stringent constraints which are in better agreement with the selection done by spectroscopy.

We note that we have selected a much lower number of physically bounded systems than Lindroos (1986). If we compare the ages derived by Lindroos (1985, 1986) for the early-type stars obtained by us, we note a very large systematic effect (Fig. 15) which explains our different conclusion.

\section{Conclusion}

The comparison of the ages of early- and late-type components of a visual binary system can, in principle, impose a strong constraint in selecting the likely physical systems, independent of any assumption except that of a common distance of the system components.

New stellar evolutionary models and more accurate distances have prompted us to perform such an analysis of the visual systems with secondaries of spectral type later than F2, selected by PPR among those suggested to be pTT candidates according to Lindroos $(1985,1986)$, the analysis by Lindroos being already based, partly, on age estimations.

For that purpose, we have derived the effective temperature for each object from photometric data, using several calibrations. in order to compute the error bar on these determinations The luminosities and their associated errors were derived by using Hipparcos parallaxes. Then ages and masses were estimated for each component by using different isochrone computations from post-main sequence and pre-main sequence evolutionary models.

The selection of possible physically bounded systems obtained with the present approach and that made by PPR on the basis of spectroscopic evidence of the young age of the late-type components are not always coincident. The presence of a strong Li I feature required by PPR is a more stringent criterion than that of coherent ages of primary and secondaries. In fact stars belonging to the selected sample may form a physically bound system even if the low-mass component has already reached an evolutionary stage so near to the ZAMS that it has lost all signatures of a pTT star. A similar remark is valid for the MMR study which used a similar approach and added the radial velocity criterion.

For stars considered by PPR as almost certainly physical systems and possible physical systems we find that most of these systems are possible physical ones according to our criterion based on their coeval age.

For the stars belonging to the third group of PPR likely optical systems our criterion detects a few possible physical systems. This different result is understandable because PPR based their results on spectroscopic criteria of youth present or absent in their spectra. We note that MMR in their analysis did not rule out some of these systems on the basis of the ages quoted by Lindroos (1986).

Physical binary systems with early-type primaries and late-type secondaries are a rich source of information on the latest phases of low mass star evolution before reaching the ZAMS and on the gas which may still surround them. It is also very informative about the formation processes of binary stars.

The extension of a study similar to the present one to a larger sample of potential binaries composed of early and late type stars will provide a first selection of a wider sample of pTT candidates to be observed spectroscopically at high resolution to search for youth signatures.

This approach allows us to reduce only to likely physical pairs the complementary spectroscopic observations required to classify the secondaries as pTT.

Acknowledgements. We would like to thank F. Palla for providing his data, tracks and isochrones to us. We are very grateful 


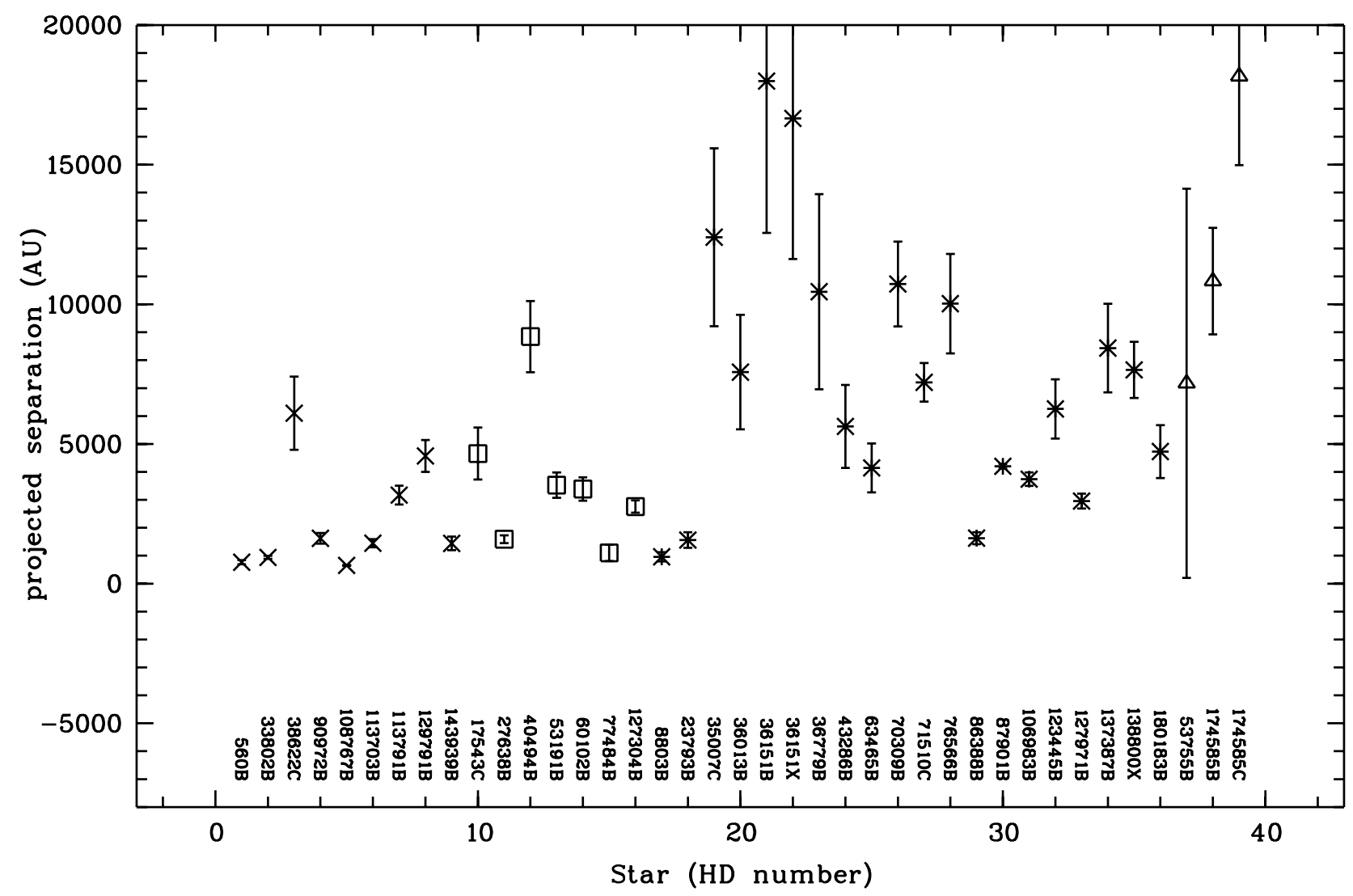

Fig. 14. The projected separation in AU versus the group of the star according to PPR. The different symbols correspond to: $(\times)$ for the first group, square for the second group, $\left(^{*}\right)$ for the third group of PPR and triangle for the MMR stars. The values of the error bars have been computed from the $\%$ error given in the last column in Table 10.

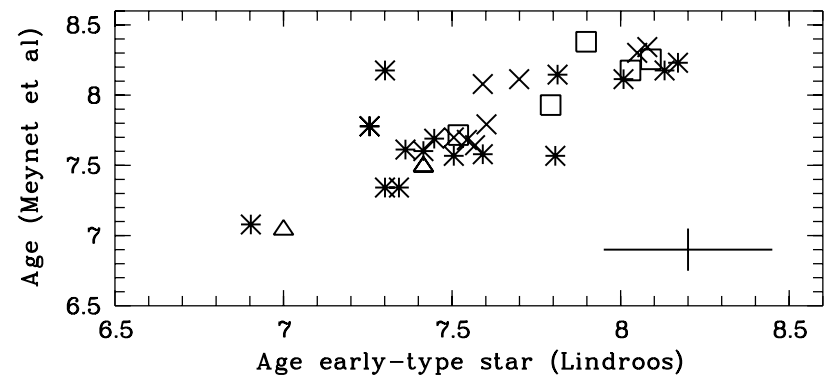

Fig. 15. The ages of the early-type stars derived by Lindroos $(1985,1986)$ compared to those computed from the Meynet et al. (1993) isochrones. The symbols are the same as in Fig. 14. Typical error bars are plotted in the lower right corner. The error on the Lindroos age estimation is taken from Lindroos (1985).

to C. A. Tout for having computed ages and masses from his set of tracks and isochrones. We are grateful to the referee Ralph Neuhäuser for his thoughtful comments which led to several improvements of this paper. This research has made use of the Simbad database, operated at CDS, Strasbourg, France. R.F. acknowledges grants from MURST $40 \%$ and $60 \%$.

\section{References}

Abt, H.A., \& Biggs, E. 1972, Bibliography of stellar radial velocities, Kitt Peak
Abt, H. A. 1988, ApJ, 331, 922

Abt, H. A., Gomez, A. E., \& Levy, S. G. 1990, ApJS, 74, 551

Alonso, A., Arribas, S., \& Martínez-Roger, C. 1996a, A\&A, 313,873

Alonso, A., Arribas, S., \& Martínez-Roger, C. 1996b, A\&AS, 117,227

Alonso, A., Arribas, S., \& Martínez-Roger, C. 1999, A\&AS, 140,261

Baraffe, I., Chabrier, G., Allard, F., \& Hauschildt, P. H. 1998, A\&A, 337, 403

Bertelli, G., Bressan, A., Chiosi, C., Fagotto, F., \& Nasi, E. 1994, A\&AS, 106, 275

Bessell, M. S. 1983, PASP, 95, 480

Bessell, M. S., Castelli, F., \& Plez, B. 1998, A\&A, 333, 231 and 337,321

Blackwell, D. E., Petford, A. D., Arribas, S., et al. 1990, A\&A, 232,396

Bright Star Catalogue, Hoffleit, D., \& Warren, W. H. 1994, 5th revised ed., version june 1994 (private communication) [BSC]

Castelli, F. 1991, A\&A, 251, 106

Claret, A., \& Gimenez, A. 1992, A\&AS, 96, 255

Claret, A. 1995, A\&AS, 109, 441

Chen, B., Vergeley, J. L., Valette, B., \& Carraro, G. 1998, A\&A, 336, 137

Cramer, N. 1993, A\&A, 269, 457

D'Antona, F., \& Mazzitelli, I. 1998, update of 1994, ApJS, 90, 467 on the Web page http://www.mporzio.astro.it/people.html 
Davidson, G. T., Claflin, E. S., \& Haisch, B. M. 1987, AJ, 94, Moon, T. T. 1985, Com. from Univ. London Obs., 78, and 771 revision 1985 (private comm.)

Duflot, M., Figon, P., \& Meyssonnier, N. 1995, A\&AS, 114, Moon, T. T., \& Dworetsky, M. M. 1985, MNRAS, 217, 305 269

ESA The Hipparcos Catalogue, 1997, ESA SP-1200 [MD]

Faraggiana, R., \& Malagnini, M. L. 1984, A\&A, 137, 149

Gahm, G. F., Ahlin, P., \& Lindroos, K. P. 1983, A\&AS, 51, 143

Girardi, L., Bressan, A., Bertelli, G., \& Chiosi, C. 2000, A\&AS, 141,371

Hauck, B., \& Mermilliod, M. 1998, A\&AS, 129, 431

Hejlesen, P. M. 1980, A\&AS, 39, 347

Herbig, G. H. 1978, Problems of Physics and Evolution of the Universe, ed. L. V. Mirzoyan (Armenian Acad. Sci., Yerevan), 171

Houdashelt, M. L., Bell, R. A., \& Sweigart, A. V. 2000, AJ, 119,1448

Huélamo, N., Neuhäuser, R., Stelzer, B., et al. 2000, A\&A, 359, 227 [HNSSZ]

Iben, I., \& Talbot, R. J. 1966, ApJ, 144, 978

Jaschek, C., Jaschek, M., \& Kucewicz, B. 1964, Zeit. f. Astr., 59,108

Koornneff, J. 1983, A\&A, 128, 84

Künzli, M., North, P., Kurucz, R. L., et al. 1997, A\&AS, 122, 51

Lada, Ch. J., \& Adams, F. C. 1992, ApJ, 393, 278

Lindroos, K. P. 1983, A\&AS, 51, 161

Lindroos, K. P. 1985, A\&AS, 60, 183

Lindroos, K. P. 1986, A\&A, 156, 223

Lutz, T. E., \& Lutz, H. 1977, AJ, 82, 431

Martín, E. L., Magazzù, A., \& Rebolo, R. 1992, A\&A, 257, 186 [MMR]

Meynet, G., Mermilliod, J.-C., \& Meader, A. 1993, A\&AS, 98, 477

Murphy, R. E. 1969, AJ, 74, 1082

Napiwotzki, R., Schönberner, D., \& Wenske, V. 1993, A\&A, 268,653

Palla, F., \& Stahler, S. W. 1999, ApJ, 525, 772

Pallavicini, R., Pasquini, L., \& Randich, S. 1992, A\&A, 261, 245 [PPR]

Papaj, J., Wegner, W., \& Krelowski, J. 1991, MNRAS, 252, 403

Pilachowski, C. A., Sneden, C., Kraft, R. P., et al. 2000, AJ, 119, 2895

Ray, P. R., Sargent, A. I., Beckwith, S. V. W., Koresko, C., \& Kelly P. 1995, ApJ, 440, L89

Richichi, A., Calamai, G., Leinert, C., et al. 1996, A\&A, 309, 163

Schaller, G., Schaerer, D., Meynet, G., \& Maeder, A. 1992, A\&AS, 96, 269

Sekiguchi, M., \& Fukugita, M. 2000, AJ, 120, 1072

Siess, L., Dufour, E., \& Forestini, M. 2000, A\&A, 358, 593

Stelzer, B., \& Huélamo, N. 2000, A\&A, 363, 667

Thé, P. S., de Winter, D., \& Pérez, M. R. 1994, A\&AS, 104, 315

Thompson, G. I., Nandy, K., \& Jamar, C. 1978, Catalogue of Stellar Ultraviolet Fluxes, The Science Research Council

Tout, C. A., Livio, M., \& Bonnell, I. A. 1999, MNRAS, 310, 360

Vergely, J. L., Freire Ferrero, R., Egret, D., \& Köppen, J. 1998, A\&A, 340, 543 